\title{
ALMA observations of cool dust in a low-metallicity starburst, SBS 0335-052
}

\author{
L. K. Hunt ${ }^{1}$, L. Testi ${ }^{1,2}$, V. Casasola ${ }^{3}$, S. García-Burillo ${ }^{4}$, F. Combes ${ }^{5}$, R. Nikutta ${ }^{6}$, P. Caselli ${ }^{7}$, C. Henkel ${ }^{8,9}$, \\ R. Maiolino ${ }^{10}$, K. M. Menten ${ }^{8}$, M. Sauvage ${ }^{11}$, and A. Weiss ${ }^{8}$ \\ 1 INAF - Osservatorio Astrofisico di Arcetri, Largo E. Fermi 5, 50125 Firenze, Italy \\ e-mail: hunt@arcetri.astro.it \\ 2 ESO, Karl Schwarzschild str. 2, 85748 Garching bei München, Germany \\ 3 INAF - Istituto di Radioastronomia \& Italian ALMA Regional Centre, via Gobetti 101, 40129 Bologna, Italy \\ 4 Observatorio Astronómico Nacional (OAN)-Observatorio de Madrid, Alfonso XII 3, 28014 Madrid, Spain \\ 5 Observatoire de Paris, LERMA (CNRS:UMR8112), 61 Av. de l'Observatoire, 75014 Paris, France \\ ${ }^{6}$ Universidad Andrés Bello, Deparamento de Ciencias Físicas, República 252, 8370134 Santiago, Chile \\ 7 School of Physics and Astronomy, University of Leeds, Leeds LS2 9JT, UK \\ ${ }^{8}$ Max-Planck-Institut für Radioastronomie, auf dem Hügel 69, 53121 Bonn, Germany \\ 9 Astronomy Department, King Abdulaziz University, PO Box 80203, Jeddah, Saudia Arabia \\ 10 Cavendish Laboratory, University of Cambridge, 19 J.J. Thomson avenue, Cambridge CB3 OHE, UK \\ 11 CEA, Laboratoire AIM, Irfu/SAp, Orme des Merisiers, 91191 Gif-sur-Yvette, France
}

Received 23 September 2013 / Accepted 21 November 2013

\section{ABSTRACT}

\begin{abstract}
We present Atacama Large Millimeter/submillimeter Array (ALMA) Cycle 0 Band 7 observations of an extremely metal-poor dwarf starburst galaxy in the Local Universe, SBS 0335-052 $(12+\log (\mathrm{O} / \mathrm{H}) \sim 7.2)$. With these observations, dust is detected at $870 \mu \mathrm{m}$ (ALMA Band 7), but $87 \%$ of the flux in this band is due to free-free emission from the starburst. We have compiled a spectral energy distribution (SED) of SBS 0335-052 that spans almost 6 orders of magnitude in wavelength and fit it with a spherical dust shell heated by a single-age stellar population; the best-fit model gives a dust mass of $(3.8 \pm 0.6) \times 10^{4} M_{\odot}$. We have also constructed a SED including Herschel archival data for IZw 18, another low-metallicity dwarf starburst $(12+\log (\mathrm{O} / \mathrm{H}) \sim 7.17)$, and fit it with a similar model to obtain a dust mass of $(3.4 \pm 1.0) \times 10^{2} M_{\odot}$. It appears that for such low-metallicity dwarf galaxies, the ratio of their stellar mass to their dust mass is within the range of values found for spirals and other star-forming galaxies. However, compared with their atomic gas mass, the dust mass of SBS 0335-052 far exceeds the prediction of a linear trend of dust-to-gas mass ratio with metallicity, while IZw 18 falls far below. We use gas scaling relations to assess a putative missing gas component in both galaxies and find that the missing, possibly molecular, gas in SBS 0335-052 is a factor of 6 times higher than the value inferred from the observed $\mathrm{H}$ I column density; in IZw 18 the missing component is much smaller. Finally, we constrain the $\mathrm{H}_{2}$ surface density conversion factor $\alpha_{\mathrm{CO}}$ with our upper limit for CO $J=3-2$ line in SBS 0335-052, and find that this is consistent with a linear or even super-linear trend of increasing $\alpha_{\mathrm{CO}}$ with decreasing metallicity. Ultimately, despite their similarly low metallicity, the differences in gas and dust column densities in SBS 0335-052 and IZw 18 suggest that metal abundance does not uniquely define star-formation processes. At some level, self-shielding and the survival of molecules may depend just as much on gas and dust column density as on metallicity. The effects of low metallicity may at least be partially compensated for by large column densities in the interstellar medium.
\end{abstract}

Key words. galaxies: starburst - galaxies: dwarf - galaxies: star formation - galaxies: ISM - galaxies: individual: SBS0335-052 galaxies: individual: IZw18

\section{Introduction}

First IRAS and ISO, then SCUBA, COBE, and most recently Spitzer and Herschel have convincingly shown that most of the star formation in the universe is enshrouded in dust. Large populations of infrared-luminous galaxies are major contributors to the far-infrared and sub-millimeter backgrounds, and are probably responsible for most of the star-formation activity at high redshifts (e.g., Chary \& Elbaz 2001; Dole et al. 2006; Gruppioni et al. 2010). Indeed, half the energy and most of the photons in the universe come from the infrared spectral region (e.g., Hauser \& Dwek 2001; Franceschini et al. 2008).

* Based on observations carried out with ALMA in Cycle 0; the Joint ALMA Observatory is operated by ESO, AUI/NRAO and NAOJ.

$\star \star$ Reduced ALMA data are only available at the CDS via anonymous ftp to cdsarc.u-strasbg. fr $(130.79 .128 .5)$ or via http://cdsarc.u-strasbg.fr/viz-bin/qcat?J/A+A/561/A49
That dust is so prominent in the high-redshift universe may appear surprising, since it has been assumed that dust would be absent in primordial, metal-poor environments. However, the presence of large amounts of dust (and molecular gas) in $z$ 6 quasars (e.g., Wang et al. 2008) has been attributed to supernovae and asymptotic giant branch stars which evolve in a metal-free interstellar medium (ISM; e.g., Todini \& Ferrara 2001; Schneider et al. 2004; Nozawa et al. 2007; Valiante et al. 2009). Dust at such high redshifts suggests that early star formation episodes must be very intense and relatively brief. However, exactly how these massive starbursts occur and evolve is not yet clear. The short interval in which star formation and the ensuing chemical enrichment and dust formation convert a dust-free metal-free environment to a dusty metal-rich one by redshift $z \sim 6$ is as yet unobserved, and studies of such transitions remain a major observational challenge. 
The Local Universe is home to star-forming dwarf galaxies that are much more metal poor than galaxies observed thus far at high redshift. Only a handful of blue compact dwarf (BCD) galaxies have been discovered so far with $12+\log (\mathrm{O} / \mathrm{H}) \sim 7.2$ (e.g., Izotov et al. 2007). Even at these extremely low metallicities, BCD spectral energy distributions (SEDs) can be dominated by infrared dust emission resulting from reprocessing of UV radiation from young massive stars (e.g., SBS 0335-052 and IZw 18: Houck et al. 2004a; Wu et al. 2007). Because such galaxies are chemically unevolved, they can provide a window on primordial galaxy formation and evolution. This is, in some sense, a "local" approach to a cosmological problem. If we can study the properties of a metal-poor ISM and its constituents locally, we may be able to better understand the high-redshift transition from metal-free Population III stars to the chemically evolved massive galaxies typical of the current epoch.

In this paper, we present Atacama Large Millimeter/submillimeter Array (ALMA) Cycle 0 Band 7 (346.21 GHz) observations of an extremely low-metallicity starburst in the Local Universe, SBS 0335-052. We also compare it with a nearby similarly metal-poor dwarf galaxy, IZw 18.

\subsection{SBS0335-052}

SBS 0335-052 (more properly, the eastern component, SBS 0335-052E) is embedded together with a western component, SBS 0335-052 W, in a giant $\mathrm{H}$ i cloud roughly 4.2 arcmin across, or $66 \mathrm{kpc}$ at SBS 0335-052's distance (Pustilnik et al. 2001; Ekta et al. 2009). SBS 0335-052 W, although physically related to SBS 0335-052 E because of their common $\mathrm{H}_{\mathrm{I}}$ envelope, is $22 \mathrm{kpc}(1.4 \mathrm{arcmin})$ distant from SBS 0335-052 E. Both galaxies are very metal-poor: $12+\log (\mathrm{O} / \mathrm{H}) \sim 7.2$ for SBS 0335-052 E and 7.12 for SBS $0335-052 \mathrm{~W}$, similar to the "prototype" BCD, IZw 18 with $12+\log (\mathrm{O} / \mathrm{H})=7.17$ (Izotov et al. 1999, 2005). SBS 0335-052 E hosts six super star clusters (SSCs), distributed (end-to-end) over roughly 2'.6 ( 700 pc), with most of the star formation activity taking place in the two brightest ones (SSC 1, 2) to the southeast (Hunt et al. 2001; Dale et al. 2001). There are more than $10000 \mathrm{O}$ stars within these two clusters, unresolved at Hubble Space Telescope (HST) resolution (Thuan et al. 1997b; Reines et al. 2008). The stars in SBS 0335-052 are mostly confined to the six SSCs, with a total mass of $\sim 6 \times 10^{6} M_{\odot}$ (Reines et al. 2008). However, there is a faint underlying stellar component with a mass comparable to the sum of the SSCs; Papaderos et al. (1998) estimate a total mass including the diffuse populations of $3.1 \times 10^{6} M_{\odot}$, roughly half of that given by Reines et al. for the clusters alone. Hence, in this paper we adopt the more massive estimate of Reines et al. (2008)

The total amount of $\mathrm{H} \mathrm{I}, \sim 4.3 \times 10^{8} M_{\odot}$ in SBS $0335-052 \mathrm{E}$ makes it extremely gas rich, even without a, as yet undetected, $\mathrm{H}_{2}$ component (Ekta et al. 2009). The global radio continuum shows a non-thermal slope (Hunt et al. 2004), but with significant free-free absorption (i.e., by dense gas) which is even more prominent at high spatial resolution. Compact rising-spectrum (optically thick) thermal radio sources are found in the vicinity of SSCs 1 and 2 (Johnson et al. 2009), together with Pa $\alpha$ (Reines et al. 2008). Even the optical spectrum implies elevated ionized gas densities $\gtrsim 500 \mathrm{~cm}^{-3}$ (Izotov et al. 1999). In the relation between star-formation rate (SFR) surface density $\Sigma_{\mathrm{SFR}}$, and gas surface density (the Schmidt-Kennicutt law), SBS 0335-052 E is the equivalent of a massive starburst, with $S F R=1.2 M_{\odot} \mathrm{yr}^{-1}$ occurring in a region of $<500 \mathrm{pc}$ in diameter. Because of their physical and apparent separation, hereafter we refer to the eastern component, SBS 0335-052E, as SBS 0335-052 without the explicit "E" suffix. Table 1 shows the basic parameters for SBS 0335-052. At the assumed distance of $\sim 54.1 \mathrm{Mpc}$, $1^{\prime \prime}=262 \mathrm{pc}$.

\subsection{Another metal-poor compact dwarf galaxy, IZw 18}

In order to place SBS 0335-052 in context, in this paper we compare its dust and gas properties with another very lowmetallicity star-forming dwarf galaxy, IZw 18.

IZw 18 is closer than SBS0335-052, at a distance of $\sim 18 \mathrm{Mpc}$, and like SBS 0335-052, is embedded together with a faint companion, the " $\mathrm{C}$ " component, in a massive $\mathrm{H}_{\mathrm{I}}$ envelope, although smaller in extent than that of SBS 0335-052 ( 16 kpc, Lelli et al. 2012). Also like SBS 0335-052, IZw 18 is extremely gas-rich, with $\sim 10^{8} M_{\odot}$ of atomic gas. Star formation in IZw 18 takes place in two massive star clusters, which together host $\sim 1900$ massive stars. These clusters are more extended than those in SBS $0335-052^{1}(\sim 150-200 \mathrm{pc}$ in diameter $)$, similar to concentrations of normal OB associations in the Milky Way and the Large Magellanic Cloud (Hunter \& Thronson 1995) ${ }^{2}$. The mass of stars in I Zw 18, 1.3 × $10^{6} M_{\odot}$ (Fumagalli et al. 2010), is about 4 times lower than in SBS 0335-052, and IZw 18's SFR, estimated from radio free-free emission (Hunt et al. 2005b) is $0.17 M_{\odot} \mathrm{yr}^{-1}$, about 5 times lower than in SBS 0335-052. The SFR surface density $\Sigma_{\text {SFR }}$ is more than 30 times lower than in SBS 0335-052. Unlike SBS 0335-052, there is no evidence for self-absorption in the radio spectrum of I Zw 18 (Hunt et al. 2005b; Cannon et al. 2005), implying the absence of dense gas.

Both SBS 0335-052 and IZw 18 are thought to be interacting systems, embedded within their common $\mathrm{H}$ i envelope (e.g., Ekta et al. 2008). This could foster star formation in massive central star clusters as modeled by Bekki (2008), perhaps causing age gradients in the stellar populations as observed (e.g., Thuan et al. 1997b; Reines et al. 2008; Contreras Ramos et al. 2011).

\subsection{Constraining cool dust in SBS0335-052}

Although Balmer lines suggest that the extinction $A_{V}$ in SBS 0335-052 is $\sim 0.5 \mathrm{mag}$ (Thuan et al. 1997b; Vanzi et al. 2000), the near-infrared Brackett line ratios $(\mathrm{Br} \gamma / \mathrm{Br} \alpha)$ imply $A_{V} \sim 12 \mathrm{mag}$ (Hunt et al. 2001). The form of the infrared (IR) SED also indicates high values $\left(A_{V} \sim 15 \mathrm{mag}\right.$, Houck et al. 2004a), but detailed studies of the optical SEDs of the individual SSCs show that the dust must be highly clumped (Reines et al. 2008). Up to now, the total dust mass was highly uncertain (Plante \& Sauvage 2002; Hunt et al. 2005a), mainly because the SED peaks at $\sim 30 \mu \mathrm{m}$ (Houck et al. 2004a), and there were no data available to constrain the mass in the cooler dust expected to dominate at longer wavelengths. Cool dust constitutes the largest contribution to the dust mass budget, so observations at wavelengths well longward of the peak of the SED are crucial for reliable estimates of dust mass.

In this paper, we add this critical information via observations of the $870 \mu \mathrm{m}$ continuum emission in this low-metallicity BCD. In Sect. 2, we present the data we have acquired and compiled in order to better constrain the dust mass and gas content in SBS 0335-052. We then estimate the free-free contribution to

\footnotetext{
Again, as throughout the paper, we are referring to SBS 0335-052E even though the suffix is omitted.

2 The conclusions of Hunter \& Thronson (1995) are still valid even with the updated distance of IZw 18.
} 
Table 1. Basic data for SBS 0335-052.

\begin{tabular}{llc}
\hline \hline Parameter & \multicolumn{1}{c}{ Value $^{a}$} & Reference $^{b}$ \\
\hline$V_{\text {hel }}$ & $4053 \mathrm{~km} \mathrm{~s}^{-1}$ & $(1)$ \\
Distance & $54.1 \mathrm{Mpc}\left(1^{\prime \prime}=262 \mathrm{pc}\right)$ & $(1)$ \\
$12+\log (\mathrm{O} / \mathrm{H})$ & 7.19 (toward SW) $-7.34(\mathrm{NE})$ & $(2)$ \\
$M_{\text {stars }}$ & $5.68 \times 10^{6} M_{\odot}$ & $(3)$ \\
$S F R$ & $1.2 M_{\odot} \mathrm{yr}^{-1}$ & $(4)$ \\
$\Sigma_{\mathrm{SFR}}{ }^{c}$ & $5 M_{\odot} \mathrm{yr}^{-1} \mathrm{kpc}^{-2}$ & This paper \\
$M_{\mathrm{HI}}$ & $4.3 \times 10^{8} M_{\odot}$ & $(5)$ \\
$\Sigma_{\mathrm{HI}}{ }^{d}$ & $56 M_{\odot} \mathrm{pc}^{-2}$ & $(6)$ \\
$L_{\text {dust }}{ }^{-2}$ & $(1.65 \pm 0.1) \times 10^{9} L_{\odot}$ & This paper \\
$M_{\text {dust }}$ & $(3.8 \pm 0.6) \times 10^{4} M_{\odot}$ & This paper \\
\hline
\end{tabular}

Notes. ${ }^{(a)}$ Luminosity and mass values taken from the literature have been scaled to the distance of $D=54.1 \mathrm{Mpc}{ }^{(b)}$ (1) NASA/IPAC Extragalactic Database (NED, http://nedwww.ipac.caltech. edu/), Luminosity Distance scaled to the reference frame defined by the 3K Microwave Background Radiation; (2) Izotov et al. (1999); (3) Reines et al. (2008); (4) Johnson et al. (2009); (5) in the entire SBS 0335-052 E region (Ekta et al. 2009); (6) Thuan \& Izotov (1997a). ${ }^{(c)}$ Obtained by considering an area of $0.2 \mathrm{kpc}^{2}$, corresponding to $\sim 1^{\prime \prime} .9 \times 11^{\prime \prime} .9 \operatorname{arcsec}^{2}$. This is a very conservative estimate, since the star formation takes place in the two brightest SSCs, unresolved at an HST resolution of $\sim 20$ pc. ${ }^{(d)}$ Obtained by integrating the SED model described in Sect. 3.

the SED, and fit the corrected multi-wavelength SED in Sect. 3 in order to estimate the total dust mass. In Sect. 4, we fit the SED of IZw 18 in the same way as for SBS 0335-052, and derive the dust mass and compare it with the gas content of I Zw 18 and with SBS 0335-052. Finally, in Sect. 5 we discuss the implied dust-to-gas ratio in SBS 0335-052 and IZw 18 and compare them with other low-metallicity dwarfs and spiral galaxies. We also constrain the CO-to- $\mathrm{H}_{2}$ conversion factor and infer limits to molecular gas mass from gas scaling relations. Our conclusions are given in Sect. 6.

\section{Data for SBS0335-052}

We have acquired Band 7 images with ALMA in Cycle 0 and also retrieved data from the Herschel archive, and combined them with data from the literature to compile a SED for SBS 0335-052 that spans almost 6 orders of magnitude in wavelength.

\subsection{ALMA observations}

The observations were carried out in five separate sessions from Jul. 30 through Aug. 14, 2012 with the ALMA observatory as part of Early Science Cycle 0. The average number of antennas was 24 and the array was in the Early Science extended configuration providing baselines up to $400 \mathrm{~m}$. SBS 0335-052 was observed with Band 7, simultaneously in the continuum and in the ${ }^{12} \mathrm{CO}(3-2)$ transition, with a rest frequency of $345.79599 \mathrm{GHz}$. The central sky frequencies of the upper and lower sidebands were $\sim 352 \mathrm{GHz}$ and $\sim 340 \mathrm{GHz}$, respectively. The observations were done as 6 executions of the same scheduling block. The unresolved extragalactic source J0339-017 was used for complex gain calibration, while Callisto or Uranus were used as flux calibrators. The measured fluxes of J0339-017 during the various observing sessions were consistently found to be $\sim 0.98 \mathrm{Jy}$ and $\sim 0.95 \mathrm{Jy}$ at $340 \mathrm{GHz}$ and $352 \mathrm{GHz}$, respectively. The flux density scale accuracy is expected to be better than $5 \%$.

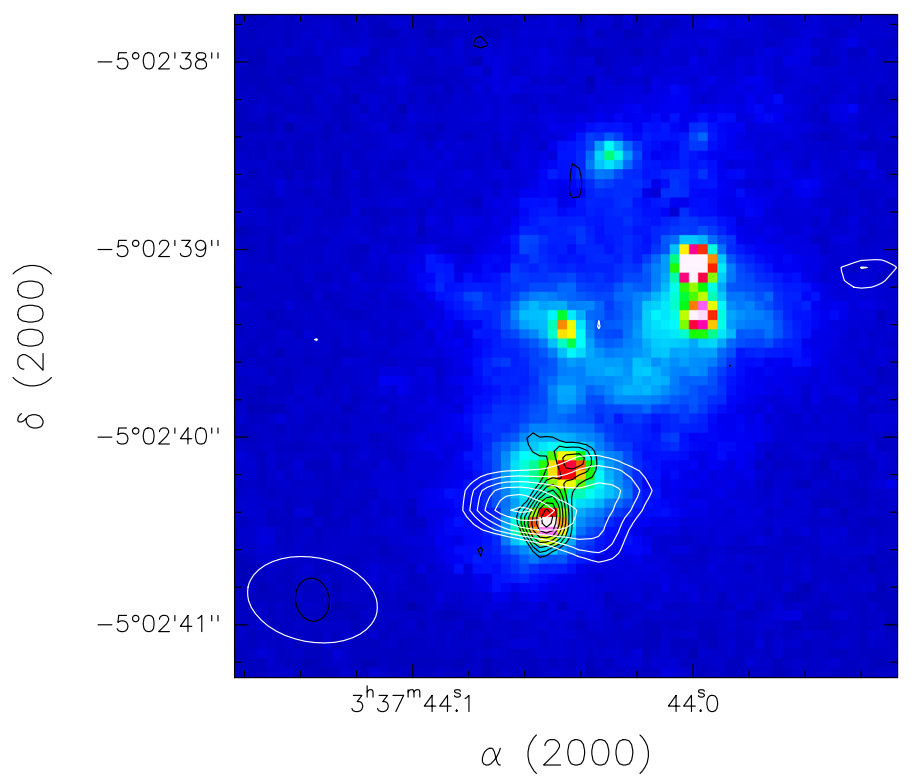

Fig. 1. ALMA $870 \mu \mathrm{m}$ continuum map of SBS0335-052 shown as white contours overlaying the HST/ACS F555M image taken from Reines et al. (2008). Band 7 contours range from 1 to $2 \sigma(\sigma=0.035 \mathrm{mJy} / \mathrm{beam})$. Black contours (ranging from 3 to $9 \sigma$, $\sigma=0.012 \mathrm{mJy} /$ beam) give the high-resolution VLA map at $3.6 \mathrm{~cm}$ taken from Johnson et al. (2009). The radio and dust emission is associated with the two brightest SSCs $(1,2)$ toward the southeast. Beam sizes of the two contoured images are shown in the lower left corner. At the distance of SBS 0335-052, 1" corresponds to $262 \mathrm{pc}$.

The full width at half maximum of the ALMA antennas' primary beam at the observing frequency is $\sim 17$ arcsec. The galaxy was observed in dual polarization mode with $1.875 \mathrm{GHz}$ total bandwidth per baseband, and a velocity resolution of $\sim 0.9 \mathrm{~km} \mathrm{~s}^{-1}$. The spectra were then smoothed to $10 \mathrm{~km} \mathrm{~s}^{-1}$ to build channel maps. The original plan was to use the line free part of the band to measure continuum emission. As no emission was detected in the $\mathrm{CO}(3-2)$ line, in the end, the full $7.5 \mathrm{GHz}$ bandwidth per polarization was used to obtain a continuum measurement centered at the average frequency of $\sim 346 \mathrm{GHz}$. The data were reduced, calibrated and imaged with the CASA software (v3.4, McMullin et al. 2007). The final maps have a beam size of $0.7 \times 0.45$ and $\mathrm{PA}$ of $79^{\circ}$, with a $1 \sigma \mathrm{rms}$ of $0.035 \mathrm{mJy} /$ beam in the continuum.

No $\mathrm{CO}(3-2)$ emission was detected. Our ALMA measurement gives a $1 \sigma$ limit of $0.8 \mathrm{mJy} /$ beam in $10 \mathrm{~km} \mathrm{~s}^{-1}$ channels, which translates to $23.5 \mathrm{mK}$. Assuming the $\mathrm{CO}$ line width in SBS 0335-052 is the same as that for $\mathrm{H}_{\mathrm{I}}\left(\sim 50 \mathrm{~km} \mathrm{~s}^{-1}\right)$, we obtain a $3 \sigma$ upper limit on the $\mathrm{CO}(3-2)$ flux of $1.58 \mathrm{~K} \mathrm{~km} \mathrm{~s}^{-1}$. Assuming an area of $1 \operatorname{arcsec}^{2}$ subtended by the source (see Sect. 5.1), and a $\mathrm{CO}(3-2) / \mathrm{CO}(1-0)$ flux ratio of 0.6 (see Sect. 5.2), this would give a $3 \sigma$ limit of the luminosity in the $\mathrm{CO}(1-0)$ line $L_{\mathrm{CO}}^{\prime}=2 \times 10^{5} \mathrm{~K} \mathrm{~km} \mathrm{~s}^{-1} \mathrm{pc}^{-2}$.

Figure 1 shows the ALMA Band 7 continuum map, with white contours superimposed on the HST/ACS F555M image from Reines et al. (2008). The black contours show the X-band Very Large Array (VLA) image $(3.6 \mathrm{~cm})$ taken from Johnson et al. (2009). The $870 \mu \mathrm{m}$ Band 7 emission is elongated in the east-west direction, and is slightly resolved (there is a possible noise excess to the northwest). The elliptical beam shape precludes a definitive analysis of the orientation and extent of the emission. In any case, like the cm-emission, the Band 7 emission is associated with the two brightest SSCs to the southeast. The peak of the ALMA continuum lies closer to SSC 1 (the 

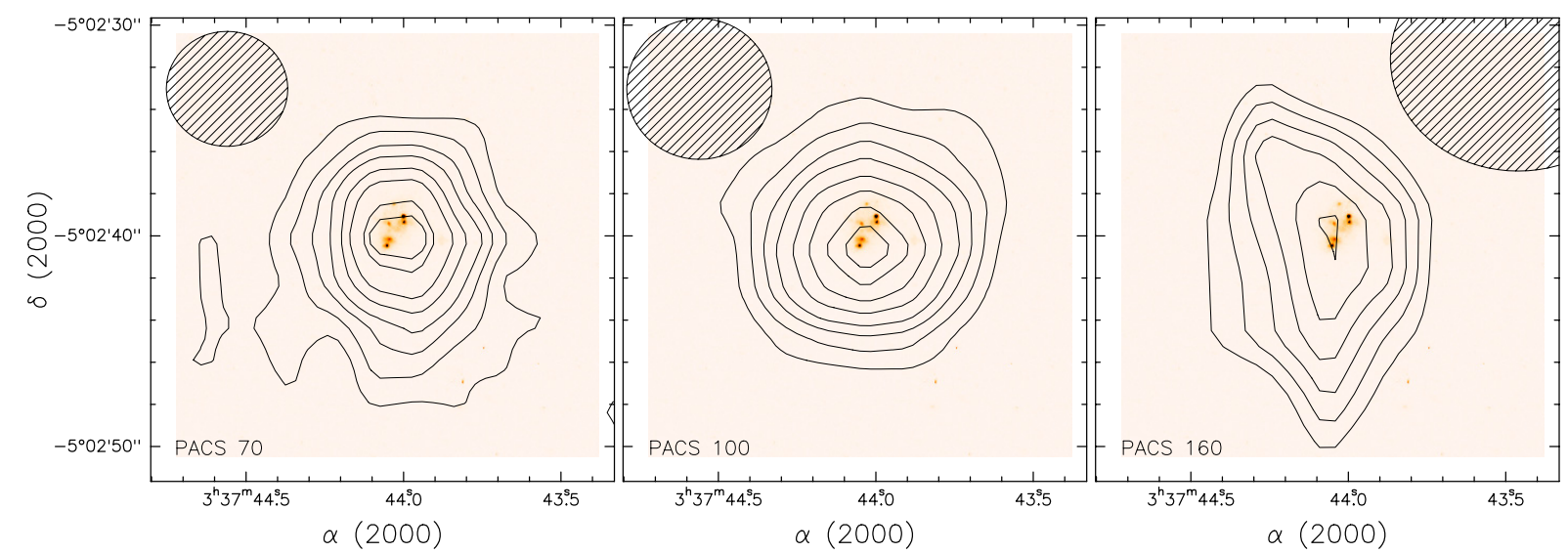

Fig. 2. PACS images of SBS 0335-052 superimposed as contours on the F555M HST/ACS image shown with false colors (see also Fig. 1). From left to right are PACS 70, 100, and $160 \mu \mathrm{m}$ maps. Contours start at $3 \sigma$ and run to $22 \sigma$ for PACS 70 , to $20 \sigma$ for PACS 100 , and for PACS 160 to $8 \sigma$. These $\sigma$ values correspond to the correlated noise measured from the images, which are roughly $4 \times$ smaller than the true noise (see PACS documentation and text). PACS beam sizes are shown as shaded ellipses in the upper portion of each panel. For display purposes, the PACS images have been rebinned to smaller pixel sizes.

southernmost cluster) than SSC 2, although slightly offset from its optical peak.

We could be missing some diffuse low-surface brightness emission in SBS 0335-052 because of the angular-size limitations of the extended ALMA configuration. The field-of-view of our map is $17^{\prime \prime}$ (only a subset of the image is shown in Fig. 1), $\sim 5 \mathrm{kpc}$, so clearly covers the entire galaxy and even some extended $\mathrm{H}_{\mathrm{I}}$ emission outside the optical confines. Nevertheless, the nature of the ALMA interferometric measurement means that emission at angular scales $\gtrsim 3^{\prime \prime}$ could be missed; such a spatial scale would be roughly the size of Fig. 1 or larger. Although we feel that this is unlikely in SBS 0335-052, additional flux at $870 \mu \mathrm{m}$ would increase the dust mass we infer in Sect. 3.

\subsection{Free-free emission at $870 \mu \mathrm{m}$ in SBS 0335-052}

$870 \mu \mathrm{m}$ emission can contain contributions from different physical processes: cool dust, thermal free-free emission (or bremsstrahlung) from ionized gas, and synchrotron emission from supernova remnants and their diffused non-thermal emission after longer timescales. In SBS 0335-052, synchrotron emission contributes a significant fraction of the total at long $\mathrm{cm}$ wavelengths and low spatial resolution (Hunt et al. 2004), but not at the highest radio frequencies observed $(43 \mathrm{GHz}$, Johnson et al. 2009). Extrapolating from the $1.3 \mathrm{~cm}$ flux of $\sim 0.52 \mathrm{mJy}$ (Hunt et al. 2004; Johnson et al. 2009), we estimate a free-free flux at $870 \mu \mathrm{m}$ of $0.40 \pm 0.07 \mathrm{mJy}$. We can also infer the freefree emission from the $\operatorname{Br} \alpha$ flux (Hunt et al. 2001), although there may be some contribution from optically-thick winds in this line (Hunt et al. 2004; Johnson et al. 2009). From $\operatorname{Br} \alpha$, we obtain a similar ALMA Band 7 flux, $0.42 \pm 0.05 \mathrm{mJy}$. Taking the average of these, $0.41 \mathrm{mJy}$, we attribute $\sim 0.06 \mathrm{mJy}$ of the total ALMA Band 7 flux (0.47 mJy) to cool dust in SBS 0335-052. By adding in quadrature the uncertainties in the Band 7 continuum level and in the radio free-free flux, we obtain the uncertainty in this dust-only $870 \mu \mathrm{m}$ flux, $\sim 0.07 \mathrm{mJy}$, implying a very marginal detection. Nevertheless, it gives us an idea of what to expect in the SED fitting discussed in Sect. 3. Taken at face value, in SBS $0335-052$ at $870 \mu \mathrm{m}$, there is a factor of 7 between the luminosity of the total emission including free-free, and that of the dust; $\gtrsim 87 \%$ of the $870 \mu \mathrm{m}$ emission is attributed to thermal free-free radiation.

\subsection{Herschel observations}

We retrieved from the Herschel Science Archive ${ }^{3}$ PACS (Photodetector Array Camera \& Spectrometer ${ }^{4}$ ) images of SBS 0335-052, acquired in guaranteed time with proposal KPGT_smadde01_1 (ObsID 1342202302, 134220303, 134220304, 134220305, 1342237748, 134223749). PACS (Poglitsch et al. 2010) observed SBS 0335-052 at 70, 100, and $160 \mu \mathrm{m}$, in large-scan mode during two visits. In the first visit, observations were acquired with 10 legs in each scan map, of $3^{\prime}$ length, separated by $4^{\prime \prime}$, repetition factor 1 which gives a total on-source exposure time of $60 \mathrm{sec}$. In the second visit, observations were acquired with scan legs of $2^{\prime}$ length, repetition factor 19 , for a total integration time of $1140 \mathrm{~s}$. In both visits at each wavelength, in order to mitigate instrumental artefacts, two different maps were acquired at $110^{\circ}$ and $70^{\circ}$, as recommended by the PACS instrument team. We reduced the observations with HIPE v.10.0 (Ott 2010), starting from the pipeline reprocessed Level 0.5 data. The "deep survey point-source" option was used, with masking performed on the images themselves before combining the repetitions and orthogonal scans into a single map. In the end, for the final maps at 100 and $160 \mu \mathrm{m}$, we used only the observations of the second visit, because of their superior signal-to-noise ratio; $70 \mu \mathrm{m}$ maps were obtained only in the first visit. Figure 2 shows the three PACS maps superimposed on the HST/ACS F555M image as in Fig. 1.

We performed photometry in apertures between radius $10^{\prime \prime}$ and $14^{\prime \prime}$, and found that the flux remains stable to within a few percent in the largest apertures; however, the noise grows with aperture size, so we used the values at the $10^{\prime \prime}$ radius. Following

\footnotetext{
3 Herschel is an ESA space observatory with science instruments provided by European-led Principal Investigator consortia and with important participation from NASA.

4 PACS has been developed by a consortium of institutes led by MPE (Germany) and including UVIE (Austria); KU Leuven, CSL, IMEC (Belgium); CEA, LAM (France); MPIA (Germany); INAFIFSI/OAA/OAP/OAT, LENS, SISSA (Italy); IAC (Spain). This development has been supported by the funding agencies BMVIT (Austria), ESA-PRODEX (Belgium), CEA/CNES (France), DLR (Germany), ASI/INAF (Italy), and CICYT/MCYT (Spain).
} 
the PACS calibration guidelines ${ }^{5}$, we adjusted the photometry for the appropriate aperture correction, and corrected the uncertainty estimates for correlated noise. Color corrections are of order unity, and within the uncertainties, so we neglected them. Uncertainties on the photometry are estimated to be $\sim 8-9 \%$ for PACS 70 and PACS 100, including calibration and uncertainties on the sky subtraction; the source is clearly detected at 70 and $100 \mu \mathrm{m}$. At $160 \mu \mathrm{m}$, the detection is roughly at $2.9 \sigma$. Our photometry is slightly higher than, but compatible within the errors to that reported by Rémy-Ruyer et al. (2013).

In order to search for extended emission, we also reduced each of the PACS images with scanamorphos (Roussel 2013), starting from the pipeline reprocessed Level 0.5 data. The photometry of the resulting images gave in fact slightly lower fluxes with larger uncertainty, perhaps because of the slightly larger reconstructed beams. Hence, we adopt the photometry as described above, and are fairly confident that the reduction technique did not miss any extended emission at any of the PACS wavelengths.

\subsection{Other multi-wavelength data}

The broadband optical, near-, and mid-infrared portions of the SED were taken from the literature (Papaderos et al. 1998; Vanzi et al. 2000; Dale et al. 2001), including data from Spitzer/IRAC (InfraRed Array Camera, Fazio et al. 2004), Spitzer/IRS (InfraRed Spectrograph, Houck et al. 2004b), and Spitzer/MIPS (Multiband Imaging Photometer, Rieke et al. 2004), taken from Houck et al. (2004a) and Engelbracht et al. (2008). For use in the SED fitting, emission features (fine-structure lines) were subtracted from the Spitzer/IRS spectrum (Houck et al. 2004a), and the spectrum was then averaged over $0.5 \mu \mathrm{m}$ bins.

Photometry from 1 to $10 \mu \mathrm{m}$ was corrected for nebular continuum emission based on the SFR, as described in Hunt et al. (2012). The SFR was converted to free-free emission by first using the conversion factor of Kennicutt (1998) to estimate $\mathrm{H} \alpha$ luminosity, then with the coefficients in Osterbrock \& Ferland (2006) to $\operatorname{Br} \alpha$ and free-free and free-bound continuum levels. We compared this method based on the SFR with the directly observed $\operatorname{Br} \alpha$ flux (Hunt et al. 2001) and the high-frequency radio emission (Hunt et al. 2004; Johnson et al. 2009) and obtain consistent results. This is an important correction since the freefree contribution at $2 \mu \mathrm{m}$ is $55 \%$, and $\sim 20 \%$ at 3.6 and $4.5 \mu \mathrm{m}$. We also included in the SED the cm-wavelength radio data from Hunt et al. (2004) and from Johnson et al. (2009). Table 2 reports the photometry described above, corrected for nebular continuum where noted.

\section{The spectral energy distribution of SBS0335-052}

We have used the multi-wavelength dataset in Table 2 to estimate the dust mass in SBS 0335-052 by fitting a spherical DUSTY model (Ivezic \& Elitzur 1997). Before fitting the SED, photometry was corrected for Galactic extinction assuming $A_{V}=0.155$ mag (taken from the NASA Extragalactic Database, $\mathrm{NED}^{6}$ ), and using the interstellar extinction curve by Cardelli et al. (1989). Because of the relatively large size of the IR beams (Spitzer, Herschel), we have considered the global photometry rather than that for only SSCs $1+2$; although the optical

\footnotetext{
5 These are found at http://herschel.esac.esa.int/twiki/ bin/view/Public/PacsCalibrationWeb\#PACS_calibration_ and_performance

6 http://ned.ipac. caltech.edu
}

Table 2. Global photometry for SBS 0335-052.

\begin{tabular}{|c|c|c|c|}
\hline $\begin{array}{l}\text { Wavelength } \\
(\mu \mathrm{m})\end{array}$ & $\begin{array}{l}\text { Flux }^{a} \\
(\mathrm{mJy})\end{array}$ & $\begin{array}{c}1 \sigma \\
\begin{array}{c}\text { Uncertainty } \\
(\mathrm{mJy})\end{array}\end{array}$ & Reference $^{b}$ \\
\hline 0.360 & 0.73 & 0.021 & (1) \\
\hline 0.440 & 0.85 & 0.013 & (1) \\
\hline 0.550 & 0.92 & 0.015 & (1) \\
\hline 0.641 & 0.81 & 0.007 & (1) \\
\hline 0.791 & 0.49 & 0.004 & (1) \\
\hline 1.25 & $0.40^{c}$ & 0.023 & (2) \\
\hline 1.65 & $0.25^{c}$ & 0.017 & (2) \\
\hline 2.20 & $0.31^{c}$ & 0.0204 & (2) \\
\hline 3.55 & $0.62^{c}$ & 0.0234 & (3) \\
\hline 4.49 & $1.50^{c}$ & 0.0493 & (3) \\
\hline 5.73 & $4.07^{c}$ & 0.1320 & (3) \\
\hline 7.87 & $12.73^{c}$ & 0.3890 & (3) \\
\hline 12.3 & 35 & 6 & (4) \\
\hline 23.7 & 79.0 & 3.12 & (3) \\
\hline 71.1 & 64.4 & 5.7 & This paper $\left(\mathrm{PACS}^{d}\right)$ \\
\hline 71.4 & 52.4 & 4.75 & (3) \\
\hline 101.2 & 31.3 & 2.7 & This paper $\left(\mathrm{PACS}^{d}\right)$ \\
\hline 162.7 & 10.4 & 3.5 & This paper $\left(\mathrm{PACS}^{d}\right)$ \\
\hline 866.5 & $0.47^{e}$ & 0.035 & This paper \\
\hline 866.5 & $0.07^{f}$ & 0.070 & This paper \\
\hline 13324 & 0.48 & 0.0789 & (5) \\
\hline 20120 & 0.40 & 0.0704 & (5) \\
\hline 35436 & 0.65 & 0.0386 & (5) \\
\hline 61685 & 0.77 & 0.0855 & (5) \\
\hline 205337 & 0.46 & 0.0610 & (5) \\
\hline 13324 & 0.56 & 0.0789 & (6) \\
\hline 20120 & 0.58 & 0.0704 & (6) \\
\hline 35436 & 0.46 & 0.0386 & (6) \\
\hline 61685 & 0.34 & 0.0855 & (6) \\
\hline
\end{tabular}

Notes. ${ }^{(a)}$ Fluxes given here are corrected for Galactic extinction, $A_{V}=0.155$ mag. ${ }^{(b)}$ (1) Papaderos et al. (1998); (2) Vanzi et al. (2000); (3) Engelbracht et al. (2008); (4) Dale et al. (2001); (5) Hunt et al. (2004); (6) Johnson et al. (2009). ${ }^{(c)}$ Corrected for nebular continuum as described in the text. (d) Photometry in an aperture of $10^{\prime \prime}$ radius. ${ }^{(e)}$ Total ALMA Band 7 continuum emission. ${ }^{(f)}$ Band 7 dust emission only, corrected for free-free contamination as described in the text.

HST data would have allowed such a separation (e.g., Reines et al. 2008), in the IR such a separation would be virtually impossible.

The DUSTY formalism solves analytically the radiative transfer problem of a spherical dust shell heated by a pointlike heating source whose immediate vicinity is devoid of dust. Thus, our model consists of two SED components: the stellar heating spectrum and a DUSTY spherical shell surrounding the internal radiation source. The dust re-processes a certain fraction of the incident radiation into the IR regime, and produces the blackbody-like peak in the total SED. The total SED is thus modeled as

$S E D_{\lambda}=a H F N_{\lambda}\left\langle\exp \left(-\tau_{\lambda}^{\text {stars }}\right)\right\rangle+b I R_{\lambda}$

where the total SED, the heating function HFN, and the IR emission are wavelength-dependent. The stars comprising the HFN will have different obscuring $\tau(V)$ toward them, so we use the average of a mix of stars and dust:

$\left\langle I_{0} \mathrm{e}^{-\tau_{\lambda}}\right\rangle=\left\langle I_{0}\right\rangle\left\langle\mathrm{e}^{-\tau_{\lambda}}\right\rangle$, 
where

$$
\left\langle\mathrm{e}^{-\tau_{\lambda}}\right\rangle=\frac{1}{\tau_{\lambda}^{\max }} \int_{0}^{\tau_{\lambda}^{\max }} \mathrm{e}^{-\tau_{\lambda}} \mathrm{d} \tau=\frac{1-\mathrm{e}^{-\tau_{\lambda}^{\max }}}{\tau_{\lambda}^{\max }}
$$

The stellar component is thus attenuated by a foreground screen of this average ( $V$-band) optical depth $\left\langle\tau_{\lambda}^{\text {stars }}\right\rangle$, not necessarily the same as that of the dust itself.

$a$ and $b$ are independently determined scales for the two components of the total SED, such that together they maximize the likelihood of the model fitting the observations. The reason behind two different normalizations is that DUSTY assumes a uniform dust cloud with a filling factor of unity. Hence, in our fits, the same DUSTY template is used to fit both stars and dust, but the relative normalization is allowed to vary in order to accommodate non-unity values of the fraction of dust that intercepts stellar light. The optical depth of the dust that reprocesses stellar radiation can be different from the optical depth of the dust that emits directly because dust may be clumpy, with the clumps having a higher opacity than the (possibly more diffuse) dust that absorbs stellar light. Once the best-fit parameters are defined, the shapes of all SED components are known, and the normalization can be found analytically (Nikutta 2012).

The optical regime of the DUSTY models requires three parameters: dust optical depth (defined in the $V$ band) for the stars, stellar age and metallicity. The input parameters for the IR portion of the DUSTY models are: the temperature at the inner part of the dust shell, $T_{\text {in }}$; the ratio of the outer shell radius to that of the inner shell boundary, $Y \equiv R_{\text {out }} / R_{\text {in }}$; the power law index $p$ of the dust distribution $\propto R^{-p}$; and the $V$-band dust optical depth $\tau_{V}^{\text {dust }}$. The DUSTY template library includes HFNs modeled as the single stellar population models of Bruzual \& Charlot (2003) at three different metallicities $\left(0.02 Z_{\odot}, 0.1 Z_{\odot}\right.$, and $Z_{\odot}$ ) and 12 different ages from 1 Myr to $10 \mathrm{Gyr}$, assuming a Chabrier (2003) Initial Mass Function. Also included in the library are three different dust grain populations: Milky-Way dust (Draine \& Li 2007), dust in the Small Magellanic Cloud (SMC; Weingartner \& Draine 2001), and chemically unevolved dust in primordial supernovae (SNe; Bianchi \& Schneider 2007).

We find the best fits and the range of model parameter values compatible with the data via exploration of the sevendimensional (7D) parameter space with an efficient Markov chain Monte Carlo code ${ }^{7}$. The three different dust-grain populations are fitted separately, and results are compared a posteriori. The HFNs and IR SEDs are tabulated on a 7D-rectilinear parameter grid, then interpolated for the specific trial parameters using the implementation from Nikutta (2012). We calculate the likelihood of each model assuming Gaussian flux errors on the measurements. The absolute normalization of the total posterior is irrelevant for the parameter estimation problem.

For most of the parameters to be fit, we assumed uniform prior probability density functions. However, we constrained the metallicity to be close to that measured by Izotov et al. (1999), $0.0347 \pm 0.014 Z_{\odot}$. We imposed a truncated Gaussian as a prior, centered at $0.0347 M_{\odot}$, with a width equal to the $1 \sigma$ uncertainty (truncated at the $1 \sigma$ limits). The final fit gives the best-fit parameters (the normalized marginalized posteriors) and their confidence intervals.

\footnotetext{
7 We based it on the PyMC package: http://pymc-devs.github. io/pymc/
}

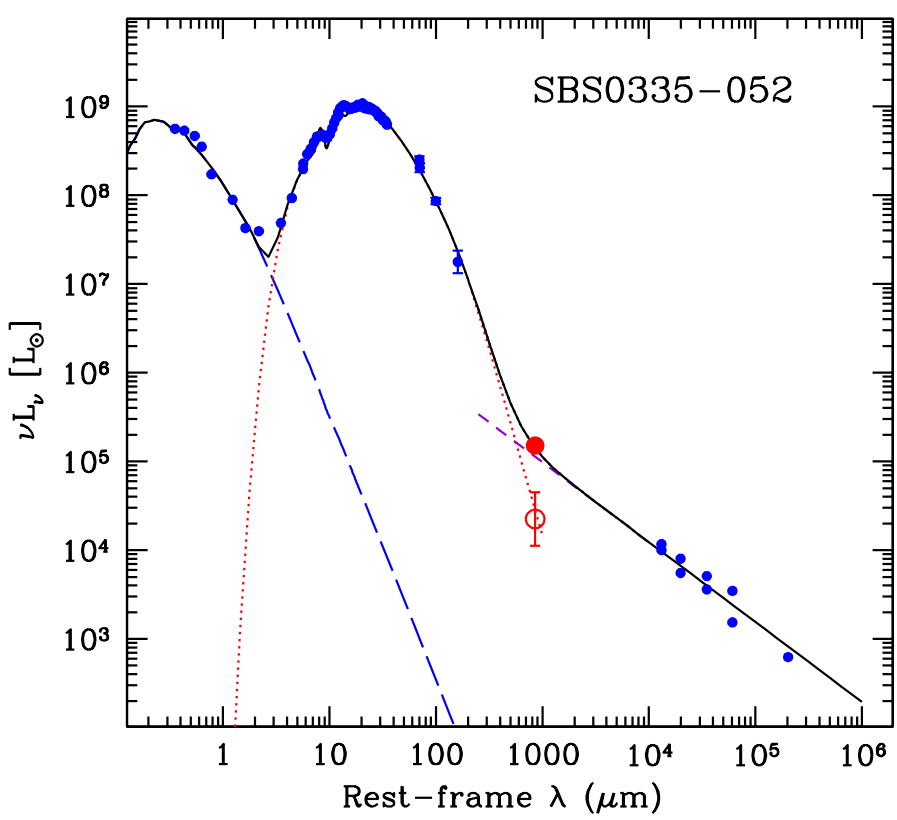

Fig. 3. SED of SBS 0335-052, together with best-fit DUSTY model overlaid. The two large (red) circles show the total ALMA Band 7 emission; the dust-only emission, estimated as described in the text, is indicated by the open (red) circle. The black curve represents the DUSTY fit, and the dotted (red) curve, only the dust; the long-dashed (blue) curve shows the stars. The radio slope is pure free-free, $\propto v^{-0.1}$, and the pure radio component is shown by a short-dashed (purple) line (longward of $250 \mu \mathrm{m}$ ). DUSTY models only extend to $1 \mathrm{~mm}$, so the excess above the dust-only emission is the extrapolation of the radio. When not evident, the error bars are masked by the size of the symbols.

\subsection{SED-fitting results}

Figure 3 shows the best-fit DUSTY model for the SED of SBS 0335-052. The radio emission has been approximated using a spectral index of $\alpha=-0.1$, appropriate for pure thermal emission. The radio emission is generally well fit by such a spectrum, although at low frequencies the spectrum is self-absorbed by the dense gas in the radio nebulae and the synchrotron component begins to be significant (Hunt et al. 2004; Johnson et al. 2009).

The SED of SBS 0335-052 is very warm; as already noted by Houck et al. (2004a), the SED peaks between 20 and $30 \mu \mathrm{m}$, even more extreme than the $60 \mu \mathrm{m}$-peaker galaxy population discovered with the Infrared Astronomical Satellite (IRAS; Vader et al. 1993). The temperature at the inner radius of the shell is $497_{-14}^{+5} \mathrm{~K}$; this relatively high temperature is necessary to fit the rapidly-rising mid-infrared portion of the spectrum. This shortwavelength peak combined with the intense star-forming event makes it difficult to detect the cool dust in the SEDs of metalpoor dwarf starbursts.

The best-fit stellar population age is $13.1_{-0.5}^{+2.8} \mathrm{Myr}$, at the lowest metallicity sampled $\left(2 \% Z_{\odot}\right)$. This age is consistent with the oldest of the SSC ages estimated by Reines et al. (2008). In fact, there is an age gradient in the clusters that could be as large as $25 \mathrm{Myr}$ from the oldest (toward the northwest) to the youngest (in the southeast, Thuan et al. 1997b), or even larger (Papaderos et al. 1998). Thus, an age of $13 \mathrm{Myr}$ seems like a reasonable compromise for our global photometry which is a luminosityweighted average. The stars themselves suffer from a total extinction $\tau_{V}^{\text {stars }}=0.5_{-2.5}^{+0.2}$, significantly smaller than the optical depth of the dust clouds (see below). The stellar mass can be inferred from the DUSTY fit, and we find $M_{\text {star }}=2.35 \times 10^{7} M_{\odot}$, 


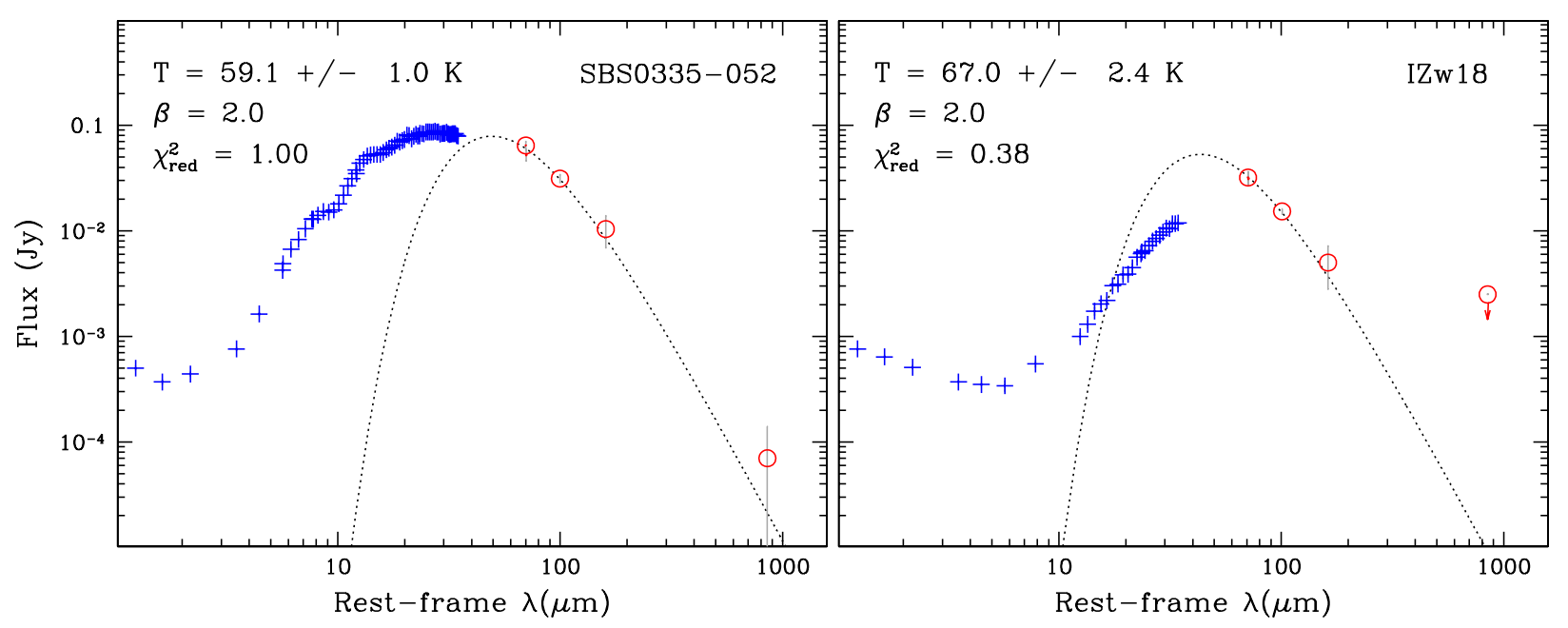

Fig. 4. Modified single-temperature blackbody fits with fixed $\beta$ of SBS 0335-052 (left panel) and IZw 18 (right panel). The blue + signs show unfitted points; the upper limit at $850 \mu \mathrm{m}$ for IZw 18 was not fit either. In the right panel, the MIPS and PACS $70 \mu \mathrm{m}$ points are so close in wavelength and measured flux that they are virtually indistinguishable. Details for IZw 18 are given in Sect. 4.

from the $V$-band luminosity of $5.6 \times 10^{8} L_{\odot}$ (corrected for extinction) and assuming a $V$-band mass-to-light ratio $\left(\mathrm{M} / \mathrm{L}_{\mathrm{V}}\right)$ of 0.04 (Bruzual \& Charlot 2003), appropriate for a 13 Myr stellar population. This is 4 times greater than the mass given by Reines et al. (2008), obtained by fitting broadband optical-nearinfrared SEDs of the 6 individual SSCs with single stellar population models (see also Fumagalli et al. 2010). However, the age found by DUSTY corresponds to the oldest cluster, which would have a $\mathrm{M} / \mathrm{L}_{\mathrm{V}}$ ratio 4 times larger than a $4 \mathrm{Myr}$ population. When we impose a prior on the stellar age (in the range of 3 to $6 \mathrm{Myr}$ ), the DUSTY fit returns a best-fit age of $4 \mathrm{Myr}$ and a $V$-band luminosity of $6.8 \times 10^{8} L_{\odot}$ (also corrected for extinction). With $M / L_{V}=0.01$, appropriate for $4 \mathrm{Myr}$, we find a stellar mass of $6.8 \times 10^{6} M_{\odot}$, only $20 \%$ more than the Reines et al. value. We thus conclude that the DUSTY stellar mass is roughly consistent with earlier estimates.

Because there is a clear silicate absorption feature at $9.7 \mu \mathrm{m}$ (Houck et al. 2004a), the dust must contain silicates; SMC dust is the grain population that provides the best fit. To reproduce this feature in absorption, it is necessary to have a relatively large quantity of cool dust in front of the massive stars heating the dust. This is naturally achieved in DUSTY through a large dust optical depth, $\tau_{\text {dust }}$, with a relatively thick shell and a large power-law index. In the case of SBS 0335-052, the bestfit $\tau_{V}^{\text {dust }}=11.8_{-2.5}^{+0.6}$; the shell relative thickness $Y=R_{\text {out }} / R_{\text {in }}$ is $605_{-21}^{+58}$, and the power-law index of the radial dust distribution $\left(\propto R^{-p}\right), p=1.63_{-0.03}^{+0.07}$. With a thinner shell and a less steep (more uniform) radial dust distribution, the silicate feature would emerge in emission rather than in absorption.

From the best-fit DUSTY model we obtain the dust mass, since the geometry of the dust and the optical properties of the grains are known. We find that there are $(3.8 \pm 0.6) \times 10^{4} M_{\odot}$ of dust in SBS 0335-052. The normalization of the DUSTY models also gives the diameter of the dust-emitting region, 2". 4 or $\sim 660 \mathrm{pc}$, comparable to the region $\left(\sim 22^{\prime \prime} 1\right)$ emitting faint $\mathrm{Pa} \alpha$ (Reines et al. 2008).

We performed two "sanity checks" for the derived dust mass: first, we performed a single-temperature modified blackbody fit (MBB) with variable emissivity, $F_{v} \propto v^{\beta} B(T, v)$, where $B(T, v)$ is the Planck function. The MBB fitting was performed by fixing $\beta$ to 2 because otherwise dust mass estimates are unreliable due to the dust opacities which are calibrated with roughly this value (Bianchi 2013). Allowed temperatures ranged from $5 \mathrm{~K}$ to $100 \mathrm{~K}$ because color corrections for PACS and MIPS are well defined only over this temperature range (e.g., Aniano et al. 2012). This fit $^{8}$, shown in Fig. 4, results in a temperature $T=59 \pm 1 \mathrm{~K}$. Using this fit at $100 \mu \mathrm{m}$ and assuming the SMC emissivity, we find a dust mass of $3.4 \times 10^{3} M_{\odot}$, a factor of 10 lower than the DUSTY value. However, Fig. 4 shows that the single- $T$ MBB fit misses a large fraction of the warmer dust, and does not pass through the ALMA point so it is very likely that a significant fraction of cooler dust is also missing. We thus conclude that the DUSTY value is not unreasonable.

As a second check, we compared the DUSTY mass with that inferred from the dust optical depth at $870 \mu \mathrm{m}$. Assuming the mean dust temperature of $\sim 59 \mathrm{~K}$ given by the MBB fit, and a mass coefficient at $870 \mu \mathrm{m}$ for SMC dust of $\kappa_{v}=4.5 \mathrm{~m}^{2} \mathrm{~kg}^{-1}$ (Weingartner \& Draine 2001), we obtain a dust mass surface density of $\Sigma_{\text {dust }}=0.45 M_{\odot} \mathrm{pc}^{-2}$. The integrated area of the region with ALMA Band 7 emission $\geq 3 \sigma$ is $\sim 1 \operatorname{arcsec}^{2}$. We would thus estimate a total dust mass of $3.1 \times 10^{4} M_{\odot}$, well within the uncertainties of the total dust mass given by the DUSTY fit. The dust mass surface density of $\sim 0.75 M_{\odot} \mathrm{pc}^{-2}$ inferred from the silicate optical depth $\tau_{9.7}(\sim 0.46$, Houck et al. 2004a) is higher than that from the $870 \mu \mathrm{m}$ dust emission. This is perhaps not surprising because the dust traced at $9.7 \mu \mathrm{m}$ in absorption is expected to be more concentrated (e.g., Dale et al. 2001).

Our estimate of the dust mass in SBS 0335-052 is more than 40 times higher than the value of $800 M_{\odot}$ given by Rémy-Ruyer et al. (2013). There are several reasons for this. The first, most obvious one, is that they use an emissivity at $100 \mu \mathrm{m}$ $\kappa_{v}=4.5 \mathrm{~m}^{2} \mathrm{~kg}^{-1}, \sim 30 \%$ higher than the best-fit SMC dust with $\kappa_{v}=3.47 \mathrm{~m}^{2} \mathrm{~kg}^{-1}$ (Weingartner \& Draine 2001). With this emissivity, Rémy-Ruyer et al. (2013) would have obtained $1037 M_{\odot}$, rather than $800 M_{\odot}$. Second, they included the $24 \mu \mathrm{m}$ point in their single-temperature MBB fit which skews fitted temperatures to higher values, thus lowering the inferred dust mass. Indeed, if we repeat our MBB fit to the fluxes in Table 2 letting

\footnotetext{
8 This fit has a $\chi_{v}^{2}$ value (1.00) not significantly worse than that performed by letting also $\beta$ vary (0.86).
} 

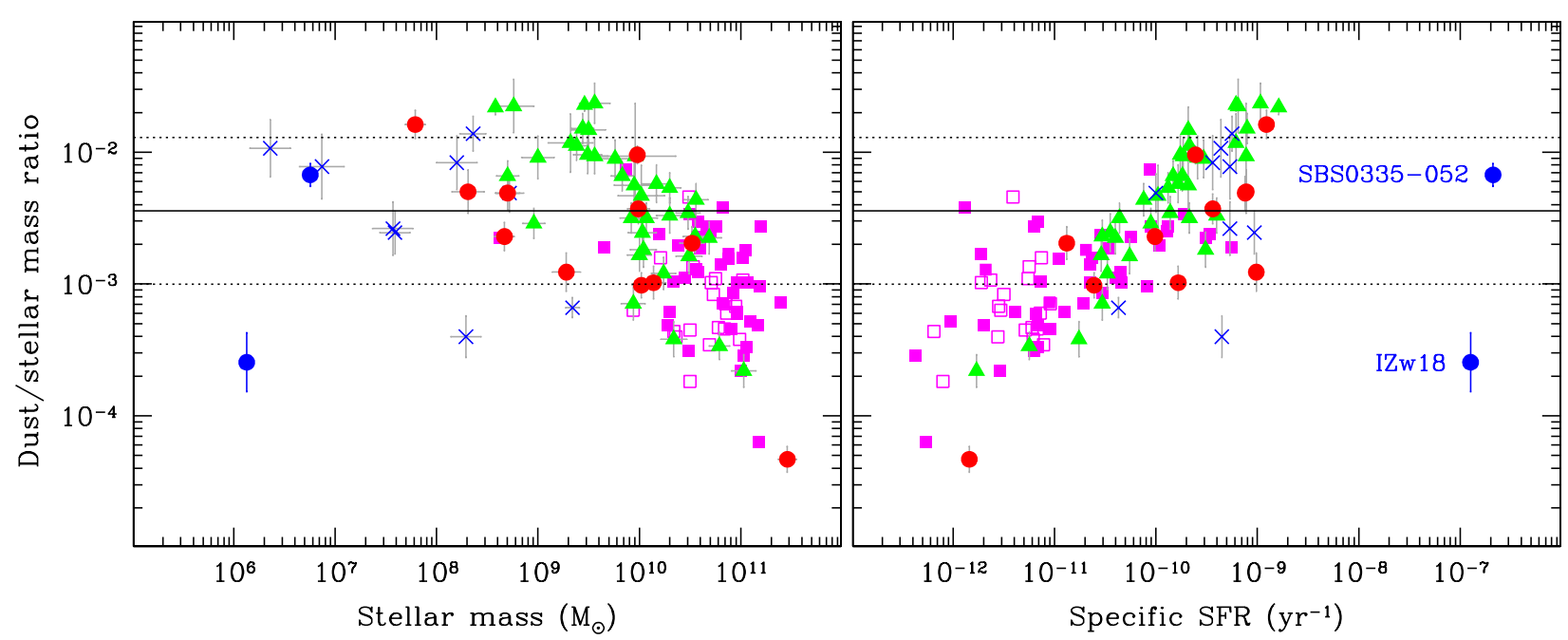

Fig. 5. Ratios of dust mass to stellar mass plotted against stellar mass $\left(M_{\text {star }}\right.$, left panel) and specific SFR (yr ${ }^{-1}$, right). SBS $0335-052$ and IZw 18 are plotted as filled (blue) circles. Data for KINGFISH galaxies, taken from Kennicutt et al. (2011), are also shown: $\times$ (for late Hubble types $T \geq 8$ ), filled (green) triangles $(0<T<8$ ), and filled (red) circles (for early Hubble types $T \leq 0$ ). The horizontal lines give the mean dust/stellar mass ratio for the KINGFISH sample, and the $1 \sigma$ standard deviation. Given as open (magenta) squares are the passive spirals studied by Rowlands et al. (2012) and as filled (magenta) squares their early-type galaxies.

$\beta$ vary and including the $24 \mu \mathrm{m}$ point, we obtain $T=87.5 \mathrm{~K}$, $\beta=1.2$, similar to $T=89_{-8}^{+10} \mathrm{~K}$ and $\beta=1.64_{-033}^{+0.39}$ given by Rémy-Ruyer et al. (2013). Our MBB fit including the $24 \mu \mathrm{m}$ point would correspond to a dust mass of $1.4 \times 10^{3} M_{\odot}$, only $35 \%$ higher than the value of Rémy-Ruyer et al. (2013) with the SMC emissivity, but less than half the value we obtain without including the $24 \mu \mathrm{m}$ point. The inclusion of the $24 \mu \mathrm{m}$ point leads to lower dust masses because of the invalidity of the assumption of a single temperature (e.g., Dale et al. 2012). In the radial temperature profile given by the DUSTY fit of SBS 0335-052, dust temperatures fall to $\sim 20 \mathrm{~K}$ in the regions farthest from the radiation source even though the innermost dust is very warm, $\sim 500 \mathrm{~K}$.

A third reason for the difference in dust masses is that the dust in SBS 0335-052 is not optically thin at short wavelengths, as implicitly assumed in the use of MBB fits. The clear silicate absorption feature and the behavior of the SED as modeled by DUSTY are signatures of the optical thickness of the dust radiation and the importance of radiative transfer. A fourth reason is that by letting $\beta$ vary in the MBB fits as done by Rémy-Ruyer et al. (2013), long-wavelength constraints become important. When we fix $\beta$ to 1.6 as in Rémy-Ruyer et al. (2013), we obtain roughly the same value of dust mass as for $\beta=1.2$ (our best-fit $\beta$ value, see above). When we let $\beta$ vary in the MBB fitting, our data result in a flatter $\beta$ slope because of the added constraint of $870 \mu \mathrm{m}$. On the other hand, when $\beta$ is fixed, the need to accommodate longer-wavelength constraints with steeper (larger values of) $\beta$ will lead to lower temperatures and consequently larger dust masses (e.g., Bianchi 2013).

The dust mass from the best-fit DUSTY model, $3.8 \times 10^{4} M_{\odot}$, gives a dust-to-stellar mass ratio of $\sim 0.007$ (assuming the stellar mass from Reines et al. 2008, see above). Ratios of dust-to-stellar mass for SBS 0335-052 are compared in Fig. 5 to the KINGFISH (Kennicutt et al. 2011) sample of galaxies and to passive spirals and early-type galaxies from $\mathrm{H}-$ ATLAS/GAMA (Rowlands et al. 2012). Also shown is IZw 18 (see Sect. 4). H-ATLAS/GAMA values are derived from opticalinfrared SED fitting. For KINGFISH, stellar parameters are taken from Kennicutt et al. (2011), or when not available, from Skibba et al. (2011) rescaled to the correct distances; dust masses are derived by Bianchi (2013). Since in these galaxies dust is usually spatially coincident with the stars, we have used global values for both sets of masses. Figure 5 shows that there is considerable scatter at low stellar masses, $M_{\text {star }}$, but for more massive galaxies with $M_{\text {star }} \approx 10^{10} M_{\odot}$, dust-to-stellar mass ratios tend to be lower than at the low-mass end. The ratio for SBS 0335-052 is within the range of the KINGFISH galaxies (see also Cortese et al. 2012).

The right panel of Fig. 5 shows the dust-to-stellar mass ratios plotted against specific SFR, the ratio of SFR to $M_{\text {star }}$ (sSFR). As found by da Cunha et al. (2010), these two quantities are well correlated. Such a correlation would be expected if dust is broadly tracing the ISM content (i.e., mostly gas with approximately the same dust-to-gas ratio, see for example Eales et al. 2012). Galaxies with higher sSFR would be in earlier evolutionary stages with a higher ISM fraction. SBS 0335-052 has a $S S F R \sim 100$ times higher than the highest SSFR in the KINGFISH sample which implies that it is in a very early phase of its evolution. The comparison with the KINGFISH and HATLAS/GAMA samples shows that the galaxies with the highest sSFRs, including SBS 0335-052, generally have high dust-tostellar mass ratios. While it would be tempting to draw a regression line between dust-to-stellar mass ratios and sSFR, models suggest that for $S S F R \gtrsim 10^{-9} \mathrm{yr}^{-1}$, the dust content falls (e.g., Calura et al. 2008; da Cunha et al. 2010). The early evolutionary stages implied by high sSFRs would presumably be in the dustformation phase of the ISM, implying that the overall dust content is lower relative to later more evolved stages of evolution.

Figure 6 plots dust-to-stellar mass ratios vs. nebular oxygen abundance, $12+\log (\mathrm{O} / \mathrm{H})^{9}$. The mass ratios are less well correlated with metallicity than with stellar mass or sSFR. SBS 0335-052 and IZw 18 (see next section) have a similarly low metallicity, being among the most metal-poor star-forming galaxies in the Local Universe, but their dust-to-stellar ratios differ dramatically; however both galaxies have ratios that are similar to those of galaxies more than 30 times more metal-rich.

\footnotetext{
9 These are the Pilyugin \& Thuan (2005) calibrations from Kennicutt et al. (2011), in order to be more comparable with the direct method used for SBS 0335-052 and IZw 18 based on electron temperature.
} 


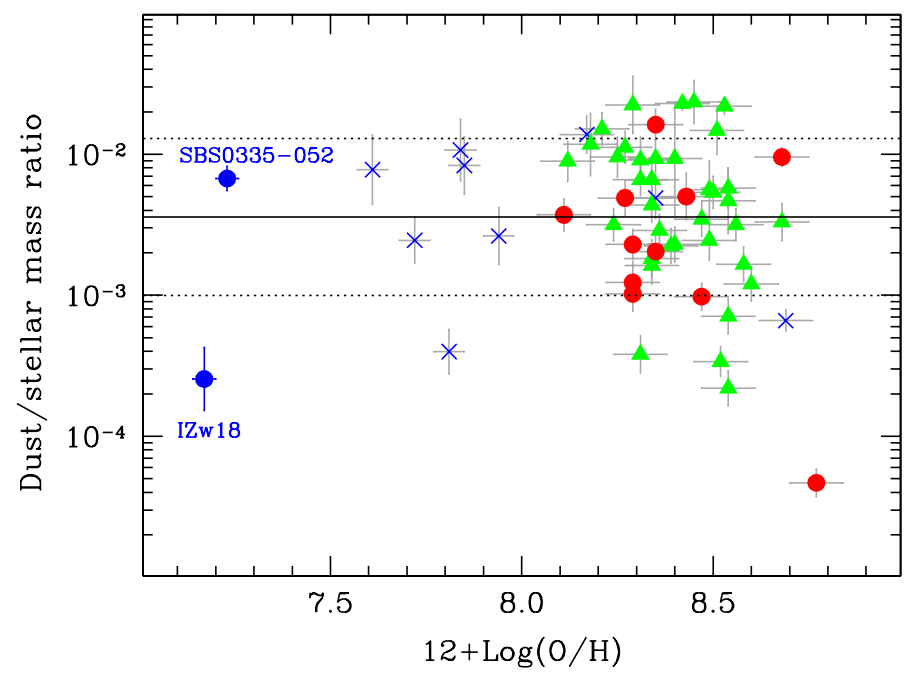

Fig. 6. Ratios of dust mass to stellar mass plotted against nebular $12+\log (\mathrm{O} / \mathrm{H})$. SBS $0335-052$ and IZw 18 are plotted as filled (blue) circles. As in Fig. 5, data for KINGFISH galaxies (see text) are also shown: $\times$ (for Hubble type $T \geq 8$ ), filled (green) triangles $(0<T<8$ ), and filled (red) circles $(T \leq 0)$. The horizontal lines give the mean dust/stellar mass ratio for the KINGFISH sample, and the $1 \sigma$ standard deviation.

Metallicity may be less important than other parameters for driving star-formation processes (e.g., Hunt et al. 2012).

\section{Comparison with $I Z w 18$}

As outlined in the Introduction, we wish to compare SBS 0335-052 with another very low-metallicity star-forming dwarf galaxy, IZw 18. Here we describe the data we have assembled for the SED of IZw 18, and the results from fitting it with the same DUSTY algorithm used for SBS 0335-052.

\subsection{Herschel data for $I Z w 18$}

We acquired from the Herschel Science Archive images of IZw 18, acquired in guaranteed time with proposal KPGT_smadde01_1 (ObsID 1342209354, 1342209355, $1342209356,1342209357)$ and in open time with proposal OT2_dfisher_1 (ObsID 1342245936, 1342245937). For 100 and $160 \mu \mathrm{m}$, we used only the latter observations as including also the former did not improve the noise characteristics of the final images. The data were reduced to Level 1 with HIPE v.10.0 (Ott 2010), starting from the pipeline reprocessed Level 0.5 data, but because of the need for conserving extended emission, we used scanamorphos (Roussel 2013) to create the final images shown in Fig. 7.

\subsection{Zw 18 photometry}

We also downloaded the Spitzer/MIPS 24, 70, and $160 \mu \mathrm{m}$ datasets, and combined the individual images into mosaics with MOPEX (Makovoz \& Marleau 2005). The emission in the $24 \mu \mathrm{m}$ image extended to radii of 12-13", which dictated the apertures we used for photometry. We used the $24 \mu \mathrm{m}$ photometry to check if the IRS spectrum was missing flux because of the $\sim 11^{\prime \prime}$ widths of the long-wavelength slits. After applying the MIPS aperture correction of 1.15 , the measured flux of $6.3 \mathrm{mJy}$ agrees very well with the $24 \mu \mathrm{m}$ flux of 6.1-6.5 mJy measured by IRS.
The MIPS and PACS photometry was performed over apertures of radius $13^{\prime \prime}, 1.6$ times the optical radius $\left(\sim 88^{\prime \prime} 1\right)$. As for SBS 0335-052, we corrected the PACS photometry and uncertainties for the aperture correction and correlated noise because of non-independent pixels. Color corrections are of order unity, so as for SBS 0335-052, we did not apply them. Uncertainties on the photometry are estimated to be $6-7 \%$ for PACS 70 and $\sim 4 \%$ for PACS 100 , including calibration and uncertainties of the sky subtraction. For MIPS photometry, we applied aperture and color corrections as described in the MIPS data reduction guide. Final uncertainties for MIPS including sky and calibration uncertainties are estimated to be $\sim 11 \%$ at $24 \mu \mathrm{m}$, and $\sim 14 \%$ at $70 \mu \mathrm{m}$, slightly higher than those quoted by Engelbracht et al. (2008) and Herrera-Camus et al. (2012), respectively. Our photometry agrees very well with these previous estimates.

IZw 18 is clearly detected at 70 (MIPS, PACS) and $100 \mu \mathrm{m}$ (PACS). At $160 \mu \mathrm{m}$ with PACS, the detection is marginal, $\sim 2.4 \sigma$ (see Fig. 7). Our photometry is consistent with that reported by Rémy-Ruyer et al. (2013) for 70 and $100 \mu \mathrm{m}$. Using shallower images, both Rémy-Ruyer et al. (2013) and Herrera-Camus et al. (2012) report non-detections at $160 \mu \mathrm{m}$ : the former give a $5 \sigma$ upper limit of $11 \mathrm{mJy}$, and the latter a $3 \sigma$ limit of $27.2 \mathrm{mJy}$, both consistent with our measurement based on deeper data.

Basic data for IZw 18 are reported in Table 3, and the compiled SED data are given in Table 4. At the assumed distance of $18.2 \mathrm{Mpc}, 1^{\prime \prime}=88 \mathrm{pc}$.

\subsection{Fitting the SED of $I Z w 18$}

As for SBS 0335-052, before fitting the SED, it was necessary to correct the photometry from $1-10 \mu \mathrm{m}$ for nebular emission. Based on IZw 18's SFR $=0.17 M_{\odot} \mathrm{yr}^{-1}$ (Hunt et al. 2005b, scaled to $D=18.2 \mathrm{Mpc}$ ), this turns out to be an important correction: $\sim 50 \%$ of the $3.6 \mu \mathrm{m}$ and $4.5 \mu \mathrm{m}$ emission is from nebular continuum $+\operatorname{Br} \alpha$. IZw 18 is extended at short IRS wavelengths (see $\mathrm{Wu}$ et al. 2007), and judging from the IRAC global photometry, the 3". 6 aperture for the IRS short-low spectral range misses $50 \%$ or more of the emission. Hence, the free-free subtraction resulted in unrealistically low fluxes, so we did not consider in the SED fitting the short-wavelength IRS fluxes $\lesssim 10 \mu \mathrm{m}$. The $2.6 \mathrm{~mm}$ flux observed by Leroy et al. (2007) is also contaminated by free-free emission; extrapolating from the thermal fraction $(47 \%$ ) of the $8.4 \mathrm{GHz}$ radio continuum (Hunt et al. 2005b; Cannon et al. 2005) gives a free-free component at $115 \mathrm{GHz}$ of $0.37 \mathrm{mJy}$. Leroy et al. (2007) estimate that the free-free flux at $115 \mathrm{GHz}$ is between 0.36 and $0.51 \mathrm{mJy}$, which means that from $35 \%$ to $48 \%$ of the observed flux [(1.06 \pm 0.35$) \mathrm{mJy}]$ is bremsstrahlung.

The Spitzer/IRS spectrum (not included in Table 4) is taken from Wu et al. (2007), and it was averaged over $1 \mu \mathrm{m}$ bins after subtracting emission lines. As for SBS 0335-052, to fit the SED we imposed only a prior on metallicity. The result of the SED fitting is shown in Fig. 8. The radio emission is approximated by a radio spectral index of $\alpha=-0.25$, slightly steeper than pure thermal emission because of a global synchrotron component (Hunt et al. 2005b).

Like the SED of SBS 0335-052, IZw 18's SED is warm; it peaks between 50 and $60 \mu \mathrm{m}$. The DUSTY fit fails to fit the estimated dust-only emission at $2.6 \mathrm{~mm}$, and the prediction of the fit is such that dust would be impossible to detect at this relatively long wavelength ${ }^{10}$. The temperature at the inner radius

10 The gradient in the radio spectral index makes the thermal/nonthermal separation in I Zw 18 problematic (Hunt et al. 2005b). 


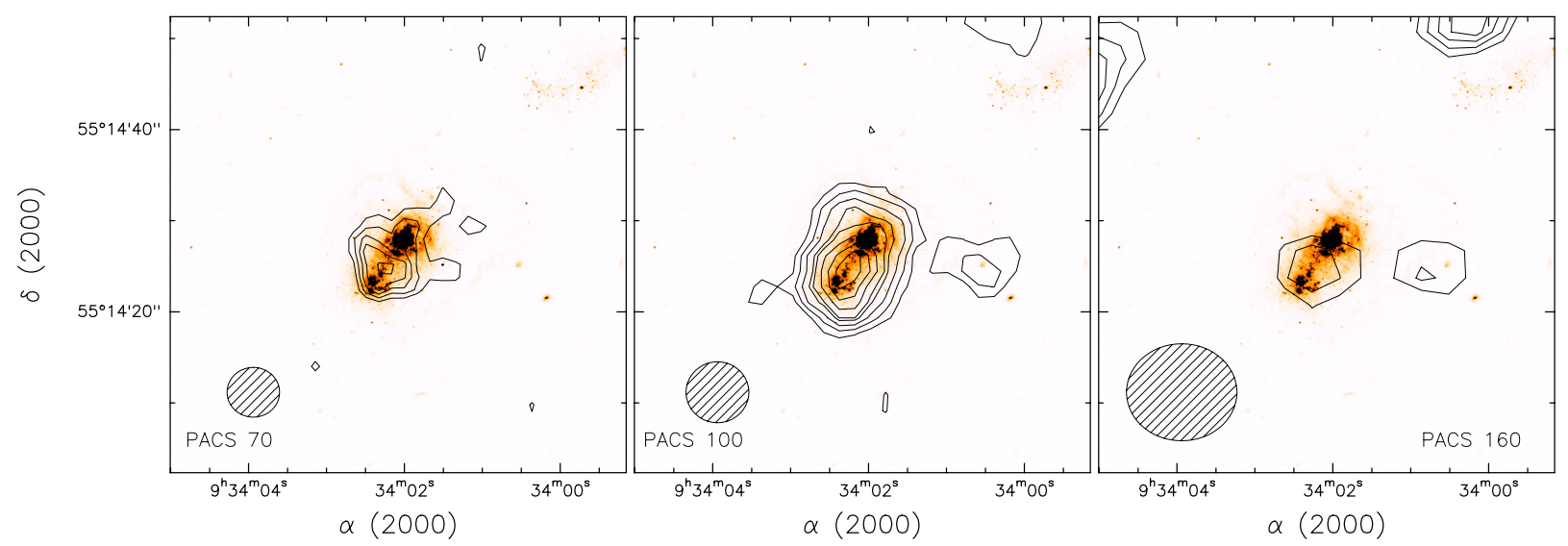

Fig. 7. PACS images of IZw 18 superimposed as contours on the F606W HST/ACS image shown with false colors. From left to right are PACS 70,100 , and $160 \mu \mathrm{m}$. Contours start at $3 \sigma$, and run to $7 \sigma$ for PACS 70, to $16 \sigma$ for PACS 100, and for PACS 160 to $2 \sigma$. As in Fig. 2 , these $\sigma$ values correspond to the correlated noise measured from the images, which are smaller than the true noise (see text).

Table 3. Basic data for I Zw 18.

\begin{tabular}{lcc}
\hline \hline Parameter & Value $^{a}$ & Reference $^{b}$ \\
\hline$V_{\text {hel }}$ & $751 \mathrm{~km} \mathrm{~s}^{-1}$ & $(1)$ \\
Distance & $18.2 \mathrm{Mpc}\left(1^{\prime \prime}=88 \mathrm{pc}\right)$ & $(2)$ \\
$12+\log (\mathrm{O} / \mathrm{H})$ & $7.17(\mathrm{NW})-7.18(\mathrm{SE})$ & $(3)$ \\
$M_{\text {stars }}$ & $1.34 \times 10^{6} M_{\odot}$ & $(4)$ \\
$S F R$ & $0.17 M_{\odot} \mathrm{yr}^{-1}$ & $(5)$ \\
$\Sigma_{\mathrm{SFR}}{ }^{c}$ & $0.19 M_{\odot} \mathrm{yr}^{-1} \mathrm{kpc}^{-2}$ & This paper \\
$M_{\mathrm{HI}}$ & $1.0 \times 10^{8} M_{\odot}$ & $(6)$ \\
$\left\langle\Sigma_{\mathrm{HI}}\right\rangle$ & $64 M_{\odot} \mathrm{pc}^{-2}$ & $(6)$ \\
Peak $\Sigma_{\mathrm{HI}}$ & $100 M_{\odot} \mathrm{pc}^{-2}$ & $(6)$ \\
$L_{\text {dust }}{ }^{d}$ & $(2.00 \pm 0.07) \times 10^{7} L_{\odot}$ & This paper \\
$M_{\text {dust }}$ & $(3.4 \pm 1.0) \times 10^{2} M_{\odot}$ & This paper \\
\hline
\end{tabular}

Notes. ${ }^{(a)}$ Luminosity and mass values taken from the literature have been scaled to the distance of $D=18.2 \mathrm{Mpc}{ }^{(b)}$ (1) NASA/IPAC Extragalactic Database (NED); (2) Aloisi et al. (2007); (3) Izotov et al. (1999); (4) Fumagalli et al. (2010); (5) Hunt et al. (2005b); (6) Lelli et al. (2012). ${ }^{(c)}$ Obtained by considering an area of $0.88 \mathrm{kpc}^{2}$, corresponding to a circular aperture $6^{\prime \prime}(\sim 530 \mathrm{pc})$ in radius. ${ }^{(d)}$ Obtained by integrating the SED model described in Sect. 4.

of the shell is $850_{-147}^{+20} \mathrm{~K}$, warmer than in SBS 0335-052; this temperature is the maximum probed by our DUSTY template library, so perhaps it is even a lower limit. The shell is about 5 times thinner than for SBS 0335-052, $Y \equiv R_{\text {out }} / R_{\text {in }} 132_{-18}^{+11}$ vs. $Y \sim 600$. Unlike SBS $0335-052$, there is no signature of silicate absorption in the SED of IZw 18; in fact the best-fitting dust is that produced by primordial Population III SNe (Bianchi \& Schneider 2007) rather than the SMC dust that gave the best fit for SBS 0335-052. It was impossible to fit the SED of IZw 18 with either the Milky Way dust or the SMC dust as given by Weingartner \& Draine (2001); this could be because of an intrinsic low silicate abundance in IZw 18 since the SNe dust used in our models (Bianchi \& Schneider 2007) contains no silicate grains.

The best-fit stellar age is $18.3_{-2.5}^{+1.7} \mathrm{Myr}, \approx 2 \%$ solar metallicity, and with no measurable extinction. This age is compatible with the mix of ages obtained from fitting the global optical+NIR photometry (Hunt et al. 2003). The implied stellar mass given by the $V$-band luminosity of $6.56 \times 10^{7} L_{\odot}$, and $M / L_{V}=0.028$ (for a stellar population $18 \mathrm{Myr}$ old, Bruzual \& Charlot 2003) is $1.82 \times 10^{6} M_{\odot}$, roughly consistent with the stellar mass of $1.34 \times 10^{6} M_{\odot}$ found by Fumagalli et al. (2010)
Table 4. Global photometry for I Zw 18.

\begin{tabular}{|c|c|c|c|}
\hline $\begin{array}{l}\text { Wavelength } \\
(\mu \mathrm{m})\end{array}$ & $\begin{array}{l}\text { Flux }^{a} \\
(\mathrm{mJy})\end{array}$ & $\begin{array}{c}1 \sigma \\
\begin{array}{c}\text { Uncertainty } \\
(\mathrm{mJy})\end{array}\end{array}$ & Reference $^{b}$ \\
\hline 0.366 & 1.56 & 0.223 & (1) \\
\hline 0.439 & 1.25 & 0.051 & (2) \\
\hline 0.440 & 1.82 & 0.250 & (1) \\
\hline 0.553 & 1.63 & 0.234 & (1) \\
\hline 0.555 & 1.31 & 0.055 & (3) \\
\hline 0.641 & 1.06 & 0.063 & (2) \\
\hline 0.815 & 0.80 & 0.035 & (3) \\
\hline 1.25 & $0.63^{c}$ & 0.028 & (3) \\
\hline 1.65 & $0.49^{c}$ & 0.025 & (3) \\
\hline 2.20 & $0.35^{c}$ & 0.019 & (3) \\
\hline 3.55 & $0.19^{c}$ & 0.012 & (4) \\
\hline 4.49 & $0.16^{c}$ & 0.012 & (4) \\
\hline 5.73 & $0.14^{c}$ & 0.038 & (4) \\
\hline 7.87 & $0.34^{c}$ & 0.037 & (4) \\
\hline 23.7 & 6.2 & 0.31 & (4) \\
\hline 23.7 & 6.3 & 0.68 & This paper $\left(\right.$ MIPS $\left.^{d}\right)$ \\
\hline 71.4 & 31.2 & 4.4 & This paper $\left(\mathrm{MIPS}^{d}\right)$ \\
\hline 71.4 & 33.6 & 1.70 & (5) \\
\hline 71.1 & 32.0 & 4.3 & This paper $\left(\mathrm{PACS}^{d}\right)$ \\
\hline 101.2 & 15.3 & 0.9 & This paper $\left(\mathrm{PACS}^{d}\right)$ \\
\hline 162.7 & 5.0 & 2.2 & This paper $\left(\mathrm{PACS}^{d}\right)$ \\
\hline 849.2 & $2.5^{e}$ & $\ldots$ & $(6)$ \\
\hline 2607 & $1.06^{f}$ & 0.35 & (7) \\
\hline 2607 & $0.56^{g}$ & 0.49 & (7) \\
\hline 35989 & 1.06 & 0.08 & (8) \\
\hline 61686 & 1.14 & 0.14 & (8) \\
\hline 199862 & 1.79 & 0.18 & (8) \\
\hline 205337 & 1.83 & 0.33 & (8) \\
\hline
\end{tabular}

Notes. ${ }^{(a)}$ Fluxes given here are corrected for Galactic extinction, $A_{V}=0.106$ mag. ${ }^{(b)}$ (1) Papaderos et al. (2002); (2) Gil de Paz et al. (2003); (3) Hunt et al. (2003); (4) Engelbracht et al. (2008); (5) HerreraCamus et al. (2012); (6) Hunt et al. (2005a); (7) Leroy et al. (2007); (8) Hunt et al. (2005b). ${ }^{(c)}$ Corrected for nebular continuum as described in the text. ${ }^{(d)}$ Photometry in an aperture of $13^{\prime \prime}$ radius. $\left.{ }^{(}\right)$) $3 \sigma$ upper limit. ${ }^{(f)}$ Total observed emission. ${ }^{(g)}$ Dust-only emission as calculated by Leroy et al. (2007), see Sect. 4.3.

using broad-band colors. The $V$-band dust optical depth $\tau_{V}^{\text {dust }}$ given by the DUSTY fit is $72_{-1}^{+8}$, quite high, typical of thick clouds. However, the relative normalizations of the two DUSTY 


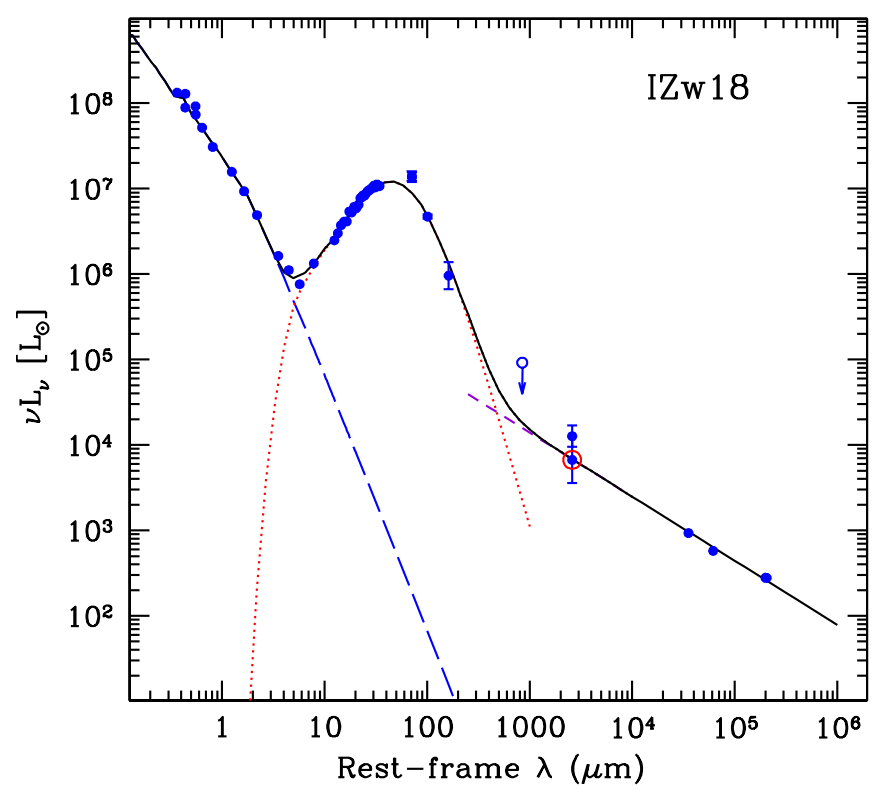

Fig. 8. SED of I Zw 18, together with best-fit DUSTY model overlaid. The large open (red) circle shows the dust-only emission taken from Leroy et al. (2007), while the small filled (blue) circle above it shows the total $2.6 \mathrm{~mm}$ emission. The black curve represents the DUSTY fit, and the dotted (red) curve, only the dust; the long-dashed (blue) curve shows the stars. The error bars are masked by the size of the symbols. As in Fig. 3, DUSTY models only extend to $1 \mathrm{~mm}$, so the excess above the dust-only emission is the extrapolation of the radio with a spectral index of -0.25 (see text). The pure radio component is shown by a short-dashed (purple) line (longward of $250 \mu \mathrm{m}$ ).

components (see Eq. (1)) implies that only $3 \%$ of the dust intercepts the stellar emission. Hence, the dust must be highly clumped.

The total dust mass in IZw 18 given by the DUSTY fit is $(3.4 \pm 1.0) \times 10^{2} M_{\odot}$, an extremely small value. However, it is roughly consistent with what would be expected compared with SBS $0335-052$. The IR luminosity of IZw $18\left(2.0 \times 10^{7} L_{\odot}\right)$ is about 80 times lower than that of SBS $0335-052\left(1.6 \times 10^{9} L_{\odot}\right.$, see Tables 1 and 3). This means for the same dust mass-to-light $(\mathrm{M} / \mathrm{L})$ ratio (i.e., for the same mean dust temperature), IZw 18 should have $\sim 1 / 80$ of the dust mass of SBS 0335-052, 475 $M_{\odot}$. Since IZw 18 has a hotter temperature as inferred from the higher value of $T_{\text {in }}$ from the DUSTY fit (and as discussed in the following), the dust mass would be even lower (because of the reduced dust M/L), thus consistent with the $\sim 340 M_{\odot}$ of dust we obtain from the DUSTY fit.

Again, as a "sanity" check, we performed a singletemperature MBB fit $^{11}$ of the SED, as shown in the right panel of Fig. 4. As for SBS 0335-052, fixing $\beta=2$ and letting $T$ vary between $5 \mathrm{~K}$ and $100 \mathrm{~K}$ in order to apply MIPS and PACS color corrections, we obtain a best-fit temperature of IZw 18 of $T=$ $67 \pm 2.4 \mathrm{~K}$, warmer than SBS 0335-052 $(T=59 \pm 1.0 \mathrm{~K})$. With the SNe dust emissivity at $100 \mu \mathrm{m}$ (a clear detection), we would estimate dust mass of $\sim 130 M_{\odot}$, a factor of $\sim 3$ lower than the DUSTY value. Judging from Fig. 4, unlike in SBS 0335-052, the single- $T$ MBB fit for I Zw 18 is missing a small fraction of dust; in fact, this fit slightly overshoots the IRS portion of the SED. Nevertheless, both the MBB and DUSTY fits may be missing some cool dust mass because of the lack of constraints at longer wavelengths $(\gtrsim 300 \mu \mathrm{m})$.

\footnotetext{
${ }^{11}$ The MBB fit assumes that the dust is optically thin, whereas the DUSTY fit would imply that the dust is somewhat optically thick.
}

There is some degeneracy in the IZw 18 DUSTY fits, in the sense that large $Y\left(R_{\text {out }} / R_{\text {in }}\right)$ and small $\tau_{\text {dust }}{ }^{12}$ give a similar SED to small $Y$ and large $\tau_{\text {dust }}$ (which is the best-fit model). Such a degeneracy is only possible in the case of featureless SNe dust, because any dust with significant silicates would produce a deep absorption feature at $\sim 10 \mu \mathrm{m}$ with large $\tau_{\text {dust }}$. The large $Y$-small $\tau_{\text {dust }}$ fits of IZw 18 give a factor of 3 less dust $\left(\sim 100 M_{\odot}\right)$ than small $Y$-large $\tau_{\text {dust }}$. The quality assessment of the small $Y$-large $\tau_{\text {dust }}$ fit of IZw 18 shows that it is statistically superior, so the larger dust mass seems more probable. Nevertheless, there is significant uncertainty in the dust mass for I Zw 18 .

Our value of $M_{\text {dust }}$ for IZw 18 is roughly consistent with the measurement by Fisher et al. (2013), given their range of acceptable values and the difference in dust emissivity for the Milky Way used by them (Draine \& Li 2007) relative to the bestfit SNe dust. At $100 \mu \mathrm{m} \kappa_{v}$ for the Milky Way is $\sim 40 \%$ lower than for the best-fit $\mathrm{SNe}$ dust, causing their lower limit of $450 M_{\odot}$ to become $326 M_{\odot}$, close to our value of $342 M_{\odot}$.

\section{Dust and gas in low-metallicity starbursts}

We have found $\sim 4 \times 10^{4} M_{\odot}$ of dust in SBS 0335-052, and an amount of dust more than 100 times lower in IZw 18. Such a result is unexpected because both BCDs have roughly the same metallicity and are equally rich in gas. The ratio of $\mathrm{H}_{\mathrm{I}}$ to stellar mass in SBS 0335-052 is 76 and 75 for IZw 18. This means that the baryonic mass is dominated by atomic hydrogen; $\mathrm{HI}$ comprises $\sim 99 \%$ of the total baryonic mass (without considering a potential molecular gas component), and will potentially provide a vast amount of fuel for future star formation.

\subsection{Dust-to-gas ratios}

The dust-to-gas mass ratio, $\mathcal{D}$, is a measure of the metals bound up in dust grains. $\mathcal{D}$ should follow the balance of dust formation processes and grain destruction from $\mathrm{SNe}$ explosions and other violent events in the ISM. If the interstellar abundances of heavy elements were proportional to the gas-phase oxygen abundance $\mathrm{O} / \mathrm{H}$, we would expect a trend of $\mathcal{D}$ with $12+\log (\mathrm{O} / \mathrm{H})$, under the assumption that the fraction of metals in dust does not vary from galaxy to galaxy. Such a correlation has been shown to hold in local galaxies (e.g., Draine et al. 2007; Galametz et al. 2011; Leroy et al. 2011) and at high redshift (e.g., Santini et al. 2010; Magdis et al. 2011).

While measurements of dust-to-gas ratios at low metallicity are becoming more frequent (e.g., Chen et al. 2013), up to now there have been no reliable estimates of dust masses at $12+\log (\mathrm{O} / \mathrm{H}) \lesssim 8$ (Draine et al. 2007; Galametz et al. 2011). Here we add SBS $0335-052$ and I Zw 18 to the available datasets, and test whether the correlation between $\mathcal{D}$ and $\mathrm{O} / \mathrm{H}$ extends to these low abundances. The main problem with such a test is that the total gas mass estimate is missing the molecular component; this point will be discussed further in Sect. 5.2. If we take the total H I mass in SBS 0335-052 from Ekta et al. (2009), integrated over a region $\gtrsim 2.5 \mathrm{kpc}^{2}$ (several $4 \times 3 \operatorname{arcsec}^{2}$ beams, see Table 1), we obtain $\mathcal{D}=8.9 \times 10^{-5}$, which would be well below the linear trend of $\mathcal{D}$ with $\mathrm{O} / \mathrm{H}$. However, as noticed by Draine et al. (2007), it is important to consider the gas mass in the same region as the dust; otherwise $\mathcal{D}$ will be unfairly underestimated. Therefore, to calculate the gas mass we relied on column densities rather than the total $\mathrm{H}_{\mathrm{I}}$ masses given in Tables 1 and 3. The dust in SBS 0335-052 subtends an area

12 This is really $\tau_{V}^{\text {dust }}$, as defined in Sect. 3 . 


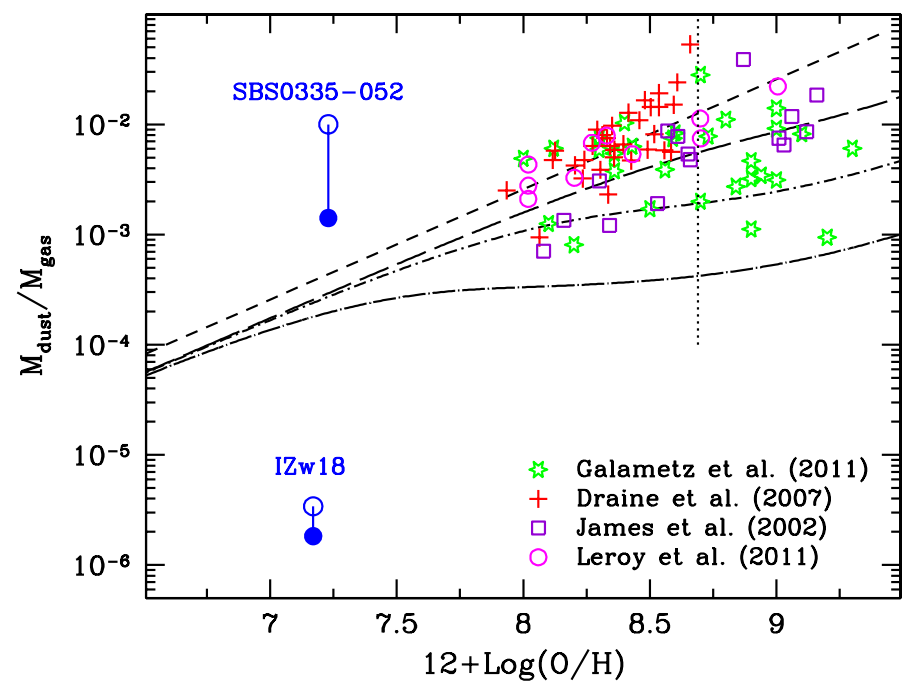

Fig. 9. Dust-to-gas mass ratios $\mathcal{D}$ as a function of metallicity. Open circles show $\mathcal{D}$ inferred from the H I gas mass of SBS 0335-052 and IZw 18; the filled (blue) circles give the total gas mass after adding the putative molecular component discussed in Sect. 5.1. Galaxies from Galametz et al. (2011) are shown as (green) open stars, those from Draine et al. (2007) as (red) +, from James et al. (2002) as (purple) squares, and from Leroy et al. (2011) as (magenta) open circles. The short-dashed line is the linear prediction by Draine et al. (2007), and the three-dot-dashed curves below show the models by Hirashita et al. (2002). The vertical dotted line illustrates solar metallicity $12+\log (\mathrm{O} / \mathrm{H})=8.69$ (Asplund et al. 2009).

of $\sim 1 \operatorname{arcsec}^{2}$, and the $\mathrm{H}_{\mathrm{I}}$ column density (measured in UV absorption) in a $2 \times 2 \operatorname{arcsec}^{2}$ aperture is $\sim 56 M_{\odot} \mathrm{pc}^{-2}$ (Thuan \& Izotov 1997a). Hence, the total H I mass in the region where dust coexists is $3.85 \times 10^{6} M_{\odot}$, and $\mathcal{D}=0.0099$. This gives the same $\mathcal{D}$ as dividing the dust column density $\left(0.55 M_{\odot} \mathrm{pc}^{-2}\right)$ by the $\mathrm{H}_{\mathrm{I}}$ column $\left(56 \mathrm{M}_{\odot} \mathrm{pc}^{-2}\right)$. Applying the same reasoning to IZw 18 , we find $\mathcal{D}=3.4 \times 10^{-6}$, assuming that both dust and gas subtend a circular region $8^{\prime \prime}(700 \mathrm{pc})$ in radius. We would obtain the same value if we divided the total dust mass $\left(3.4 \times 10^{2} M_{\odot}\right)$ by the total $\mathrm{H}_{\mathrm{I}}$ mass $\left(1 \times 10^{8} M_{\odot}\right)$, implying mean dust and $\mathrm{H}_{\mathrm{I}}$ column densities in IZw 18 of $2.2 \times 10^{-4} M_{\odot} \mathrm{pc}^{-2}$ and $64 M_{\odot} \mathrm{pc}^{-2}$, respectively.

Figure 9 plots dust-to-gas mass ratios for four samples of galaxies taken from the literature, together with our new estimates for SBS 0335-052 and IZw 18. The linear trend by Draine et al. (2007) shown as a short-dashed line fits the metalrich data quite well. However, with $\mathrm{H}_{\mathrm{I}}$ alone (shown as open blue circles in Fig. 9), SBS 0335-052 exceeds the trend by a factor of 30 or more, but IZw 18 lies almost a factor of 100 below the trend. If our estimate of dust mass in I Zw 18 is correct, at low metallicity the trend of abundance and dust-to-gas ratios changes drastically, and in different ways according to some other parameter, for example SFR surface density or column densities of dust and ionized gas.

Our ALMA observations did not detect $\mathrm{CO}(3-2)$ in SBS 0335-052, but either the expected linearity in $\mathcal{D}$ is incorrect, or there is a large fraction of missing molecular gas. We can use gas scaling relations to estimate the total gas content in SBS 0335-052 and check whether we are also missing a molecular component in IZw 18. If the SF in SBS 0335-052 occurs in the roughly same area as the ALMA emission $\left(\sim 1 \operatorname{arcsec}^{2}\right)$, we would find $\Sigma_{\mathrm{SFR}} \sim 18 \mathrm{M}_{\odot} \mathrm{kpc}^{-2}$. However, if we conservatively consider a larger area of diameter $550 \mathrm{pc}(\sim 2$ '. 1$)$, comparable to the region emitting faint $\operatorname{Pa} \alpha$ (Reines et al. 2008), we

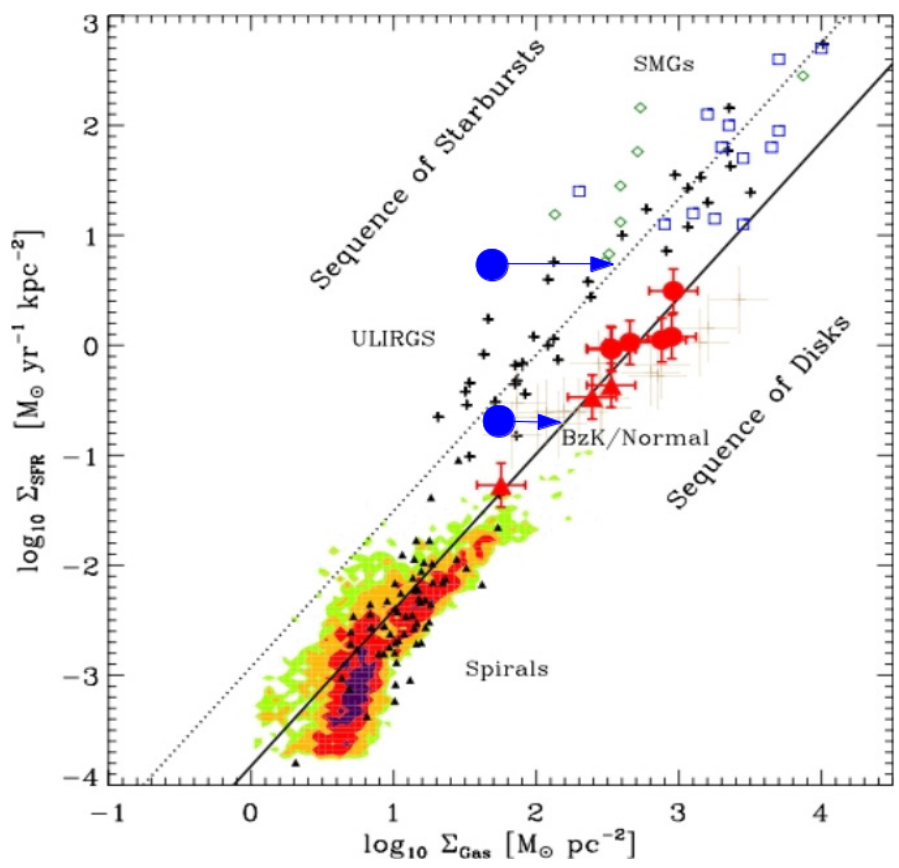

Fig. 10. SBS 0335-052 (upper filled blue circle) and IZw 18 (lower filled blue circle) on the gas scaling relations given by Daddi et al. (2010). Only H I mass density is considered for the BCDs studied here; the arrows correspond to the missing gas mass surface density that would transport SBS 0335-052 to the "starburst sequence" and I Zw 18 to the "sequence of disks". SFR surface density is plotted as a function of the gas (atomic and molecular) surface density for $z \sim 0.5$ disks (shown as red filled circles and triangles; brown crosses indicate $z=1-2.3$ normal galaxies, and empty squares correspond to sub-millimeter galaxies. The shaded regions and filled triangles are local spiral galaxies, and small crosses are starbursts and ultra-luminous galaxies. References for these data are given in Daddi et al. (2010).

obtain $\Sigma_{\mathrm{SFR}} \sim 5 M_{\odot} \mathrm{kpc}^{-2}$. The SF in IZw 18 takes place in a region of diameter $\sim 1 \mathrm{kpc}$ (Cannon et al. 2002, 2005), and we find $\Sigma_{\mathrm{SFR}} \sim 0.15 M_{\odot} \mathrm{kpc}^{-2}$. These spatial scales are sufficiently large that the gas scaling relations are not expected to have broken down (e.g., Verley et al. 2010; Onodera et al. 2010). Figure 10 shows SFR surface density plotted against gas surface density; the figure is taken from Daddi et al. (2010). SBS 0335-052 (the upper point) and IZw 18 (the lower one) correspond to filled blue circles, and the arrows indicate how much gas would be needed to bring SBS 0335-052 to the starburst sequence and IZw 18 to the sequence of disks. At SBS 0335-052's SFR surface density, the starburst sequence of the SFR-gas scaling would require a total gas surface density of $\sim 400 M_{\odot} \mathrm{pc}^{-2}$. I Zw 18 already has too much $\mathrm{H}_{\mathrm{I}}$ to fall on the starburst sequence; if, on the other hand, it were to fall on the disk sequence, it would need a total gas surface density of $\sim 158 M_{\odot} \mathrm{pc}^{-2}$. The missing gas mass, which we take as the missing molecular component, is $\sim 342 M_{\odot} \mathrm{pc}^{-2}$ for SBS 0335-052, and $\sim 94 M_{\odot} \mathrm{pc}^{-2}$ for I Zw 18, assuming for the latter the mean H column density of $64 M_{\odot} \mathrm{pc}^{-2}$ (see Tables 1 and 3). However, if I Zw 18 falls on the starburst sequence, similarly to what we hypothesize for SBS 0335-052, it would be missing no molecular mass; indeed, additional gas would move I Zw 18 farther from what we might expect. In any case, these estimates of missing molecular gas for both galaxies are highly uncertain, and should be taken as a basis for discussion, rather than conclusive determinations.

Adding the (putative) missing molecular gas gives a total gas mass that corresponds to the filled circles in the dust-to-gas mass 
ratios plotted in Fig. 9. The missing molecular component would be $\sim 1.5$ times the $\mathrm{H}_{\text {I }}$ mass in I Zw 18 but a factor of 6 times the H I mass in SBS 0335-052 (missing $342 M_{\odot} \mathrm{pc}^{-2}$ vs. $56 M_{\odot} \mathrm{pc}^{-2}$ in $\mathrm{H}_{\mathrm{I}}$ ). SBS 0335-052 would be moved closer to, and possibly consistently with, the linear trend of $\mathcal{D}$ with metallicity, while IZw 18 would be moved farther away.

We therefore tentatively conclude that there is a large component of missing molecular gas in SBS 0335-052; in IZw 18 such a conclusion seems more remote, though possible. Krumholz (2012) argues that at low metallicities (perhaps lower than in these galaxies), star formation will occur in the cold atomic phase of the ISM rather than necessarily in a molecular phase. This could be the case in SBS 0335-052 and IZw 18 since in both galaxies star formation is taking place in a very H Irich medium. Nevertheless, the total gas fractions could be even higher in SBS 0335-052; if SBS 0335-052 is to fall on the linear trend of $\mathcal{D}$ with $\mathrm{O} / \mathrm{H}$, it would be missing a yet unmeasured gas component. We discuss such a gas component in the next section.

\subsection{Constraints on molecular gas conversion factors in SBS 0335-052 and I ZW 18}

Previous attempts to detect ${ }^{12} \mathrm{CO}(1-0)$ and ${ }^{12} \mathrm{CO}(2-1)^{13}$ in SBS 0335-052 were carried out by Dale et al. (2001) at Owens Valley. After $20.6 \mathrm{~h}$ of on-source integration time at $3 \mathrm{~mm}$ and $6.6 \mathrm{~h}$ of good integration time at $1 \mathrm{~mm}$, they achieved $3 \sigma$ upper limits of $1.2 \mathrm{Jy} \mathrm{km} \mathrm{s}^{-1}$ and $3.0 \mathrm{Jy} \mathrm{km} \mathrm{s}^{-1}$, respectively. Converting these to brightness temperatures gives $3 \sigma$ flux upper limits of $5.6 \mathrm{~K} \mathrm{~km} \mathrm{~s}^{-1}$ for $\mathrm{CO}(1-0)$ and $12.2 \mathrm{~K} \mathrm{~km} \mathrm{~s}^{-1}$ for $\mathrm{CO}(2-1)$. We can use these limits to constrain values for $\alpha_{\mathrm{CO}}$, the factor to convert observed $\mathrm{CO}$ intensity $I_{\mathrm{CO}}$ to $\mathrm{H}_{2}$ column density. In order to account for the "missing" molecular gas density of $342 M_{\odot} \mathrm{pc}^{-2}$ in SBS $0335-052, \alpha_{\mathrm{CO}}$ would be $\gtrsim 61 M_{\odot} \mathrm{pc}^{-2}\left(\mathrm{~K} \mathrm{~km} \mathrm{~s}^{-1}\right)^{-1}$ for $\mathrm{CO}(1-0), 10-12$ times higher than typical values for the Milky Way (e.g., Bolatto et al. 2013), but lower than would be expected ( 30 times higher) for a linear trend of $\alpha_{\mathrm{CO}}$ with metallicity (e.g., Draine et al. 2007). Assuming a $\mathrm{CO}(2-1) / \mathrm{CO}(1-0)$ ratio of 0.7 (Leroy et al. 2011), we would obtain $\alpha_{\mathrm{CO}} \gtrsim 40 M_{\odot} \mathrm{pc}^{-2}\left(\mathrm{~K} \mathrm{~km} \mathrm{~s}^{-1}\right)^{-1}$ for $\mathrm{CO}(2-1)$.

More stringent lower limits can be achieved if we use to estimate $\alpha_{\mathrm{CO}}$ the $3 \sigma \mathrm{CO}(3-2)$ upper limit for SBS 0335-052 $\left(1.58 \mathrm{~K} \mathrm{~km} \mathrm{~s}^{-1}\right)$ given in Sect. 2. Because $\Sigma_{\mathrm{H} 2}=\alpha_{\mathrm{CO}} I_{\mathrm{CO}(1-0)}$, we need first to convert the $\mathrm{CO}(3-2)$ limit to one on $\mathrm{CO}(1-0)$. For a sample of dwarf starbursts, Meier et al. (2001) found an error-weighted mean $\mathrm{CO}(3-2) / \mathrm{CO}(1-0)$ flux ratio of $0.6 \pm 0.06$, roughly consistent with the median value of 0.7 obtained by Mao et al. (2010). Converting $\mathrm{CO}(3-2)$ to $\mathrm{CO}(1-0)$ using the range in these ratios, to accommodate the "missing" $342 M_{\odot} \mathrm{pc}^{-2}$ gas density in SBS 0335-052, $\alpha_{\mathrm{CO}} \gtrsim 130-152 M_{\odot} \mathrm{pc}^{-2}\left(\mathrm{~K} \mathrm{~km} \mathrm{~s}^{-1}\right)^{-1}$.

The same exercise can be performed for IZw 18, using the $3 \sigma \mathrm{CO}(1-0)$ limit of $0.75 \mathrm{~K} \mathrm{~km} \mathrm{~s}^{-1}$ given by Leroy et al. $(2007)^{14}$. From Fig. 10, we estimate that IZw 18 is missing $\sim 94 M_{\odot} \mathrm{pc}^{-2}$ of gas density; hence $\alpha_{\mathrm{CO}} \gtrsim 125 M_{\odot} \mathrm{pc}^{-2}\left(\mathrm{~K} \mathrm{~km} \mathrm{~s}^{-1}\right)^{-1}$. The two lower limits for SBS 0335-052 and IZw 18 are similar, but this is merely fortuitous, since it depends on the sensitivity of the $\mathrm{CO}$ observations, the missing gas, and the flux ratio of $\mathrm{CO}(3-2) / \mathrm{CO}(1-0)$ in the case of SBS 0335-052. In the case of SBS 0335-052, the

\footnotetext{
${ }^{13}$ Hereafter referred to without the isotopic prefix.

${ }^{14}$ They find a $4 \sigma$ upper limit of $1 \mathrm{~K} \mathrm{~km} \mathrm{~s}^{-1}$ which we convert to $3 \sigma$ for consistency with our SBS 0335-052 calculation.
}

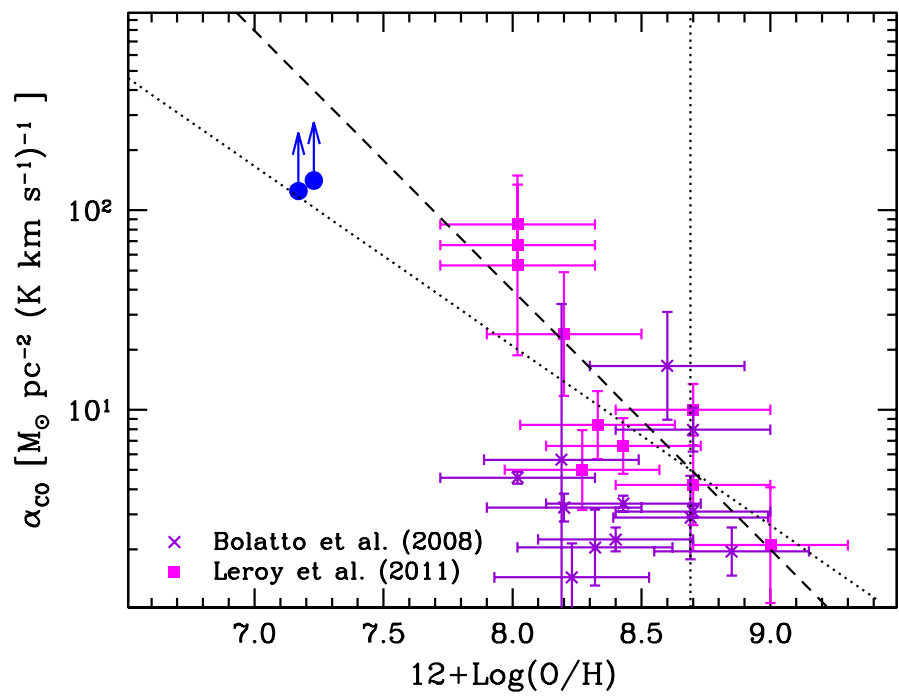

Fig. 11. $\alpha_{\text {CO }}$ vs. $12+\log (\mathrm{O} / \mathrm{H})$ for nearby galaxies. The lower limits for SBS 0335-052 and IZw 18 are shown as filled blue circles; data from Leroy et al. (2011) and Bolatto et al. (2008) are shown as filled squares and $\times$, respectively. The steeper regression, indicated by a dashed line, has a slope of -1.3 (Genzel et al. 2012) and the shallower one, shown by a dotted line, a slope of -0.9 (Sargent et al. 2013).

most conservative hypothesis would be that more gas is needed to bring it onto the starburst sequence of gas scaling relations (rather than the quiescent sequence). However, I Zw 18 is already on the starburst sequence with only $\mathrm{H}$; ; putative missing gas would be needed to move this galaxy onto the more quiescent sequence of disks. If no molecular gas component were needed for I Zw 18 in the gas scaling relations (i.e., if it really should be on the starburst sequence), $\alpha_{\mathrm{CO}}$ for I Zw 18 would be undefined.

Figure 11 shows the $\alpha_{\mathrm{CO}}$ lower limits for SBS 0335-052 and IZw 18, together with measured $\alpha_{\mathrm{CO}}$ conversion factors for two samples of nearby galaxies (Bolatto et al. 2008; Leroy et al. 2011). The steeper of the two regressions (slope -1.3) is given by Genzel et al. (2012), and the shallower one (slope -0.9 ) by Sargent et al. (2013). Our lower limits on $\alpha_{\mathrm{CO}}$ for SBS 0335-052 and IZw 18 lie slightly above the shallower regression, which would imply that the slope is closer to linear, or even superlinear as found by Genzel et al. (2012). To better constrain the low-metallicity end of the $\alpha_{\mathrm{CO}}$ conversion factor, deeper observations are needed. Even non-detections, but at a fainter intensity level, will help understand the fate of molecular gas and its tracers at low metallicity.

\section{Summary and conclusions}

We have presented our first ALMA results for SBS 0335-052, one of the most metal-poor star-forming dwarf galaxies in the Local Universe. The ALMA Band 7 continuum observations at $870 \mu \mathrm{m}$ allow us to reach an unprecedented spatial resolution of $90 \mathrm{pc}$, even with the Cycle 0 capabilities. Extrapolating from high-resolution high-frequency radio observations, we find that $85 \%$ of the Band 7 flux in SBS 0335-052 can be attributed to free-free emission. The remainder is attributed to dust, and we use this new constraint to estimate the dust mass in SBS 0335-052 by fitting its SED with a DUSTY model. The ratio of the resulting dust mass of $3.8 \times 10^{4} M_{\odot}$ in SBS $0335-052$ and its stellar mass falls within the range of normal galaxies. However, comparing the dust with $\mathrm{H}_{\mathrm{I}}$ mass and surface density, 
we find that SBS 0335-052 far exceeds the linear trend of dustto-gas mass ratio $\mathcal{D}$ with oxygen abundance.

We have compared the results for SBS 0335-052 to another dwarf galaxy at similarly low metallicity, IZw 18 . With data from the literature, and Herschel archival observations, we have estimated the dust mass in IZw 18 by fitting a DUSTY model, and find $3.4 \times 10^{2} M_{\odot}$ of dust. Compared with its $\mathrm{H}_{\mathrm{I}}$ mass and column density, unlike SBS 0335-052, IZw 18 falls far below the expected scatter of the linear trend of $\mathcal{D}$ with $\mathrm{O} / \mathrm{H}$. Finally, we have used the $\mathrm{CO}$ non-detections for both galaxies to constrain the integrated $\mathrm{CO}(1-0)$ intensity to $\mathrm{H}_{2}$ surface density conversion factor, $\alpha_{\mathrm{CO}}$, and find that the lower limits are consistent with a linear, or perhaps even super-linear trend with metallicity.

Perhaps the most significant of our findings is that metallicity does not uniquely define star-formation processes. Considering only $\mathrm{H}$ I gas, IZw 18 falls roughly on the starburst sequence of gas scaling relations, but SBS 0335-052 is a clear outlier; a gas surface density 6 times higher than that from $\mathrm{H}_{\mathrm{I}}$ alone would be required to bring it onto the sequence of starbursts (see Fig. 10).

Although specific SFRs $\left(\mathrm{SFR} / M_{\text {star }}\right)$ are similar for both galaxies $\left(\sim 10^{-7} \mathrm{yr}^{-1}\right)$, the surface densities of SF are very different. SF in SBS 0335-052 occurs mainly in two luminous compact super-star clusters, while that in IZw 18 originates in more diffuse ones; the SFR surface densities differ by more than an order of magnitude. This is probably the reason for the extreme short-wavelength peak in SBS 0335-052's SED $(\sim 20-30 \mu \mathrm{m})$ and implies that physical conditions are extreme in this galaxy. Considering also the large volume densities of ionized gas implied by its self-absorbed thermal radio spectrum (Hunt et al. 2004; Johnson et al. 2009), the star formation activity in SBS 0335-052 must be occurring in very warm, dense regions. The physical conditions in I Zw 18 are less extreme; the radio spectrum is not self-absorbed, and electron densities are lower.

Ultimately, the influence of metallicity may not be as clear cut as commonly thought. At some level, self-shielding and the survival of molecules may depend just as much on gas and dust column density as on metallicity. The effects of low metallicity may at least be partially compensated for by large column densities in the ISM. More observations of extreme metal-poor galaxies are needed to judge whether this is generally true, and to better understand the degree to which such galaxies can serve as proxies for galaxy formation at high redshift.

Acknowledgements. We appreciate the prompt review and careful comments of the referee which improved the clarity of the paper. We warmly thank Bruce Draine for constructive comments and suggestions, and Emanuele Daddi for interesting discussions. This paper makes use of the following ALMA data: ADS/JAO.ALMA\#2011.0.00039.S (PI Hunt). ALMA is a partnership of ESO (representing its member states), NSF (USA) and NINS (Japan), together with NRC (Canada) and NSC and ASIAA (Taiwan), in cooperation with the Republic of Chile. The Joint ALMA Observatory is operated by ESO, AUI/NRAO and NAOJ. We are grateful to the Italian Alma Regional Center for assistance with data handling and analysis, and to the International Space Science Institute for hospitality during the conception of the paper. L.K.H. acknowledges support from PRIN-INAF 2012/13. We made use of the NASA/IPAC Extragalactic Database (NED).

\section{References}

Aloisi, A., Clementini, G., Tosi, M., et al. 2007, ApJ, 667, L151 Aniano, G., Draine, B. T., Calzetti, D., et al. 2012, ApJ, 756, 138 Asplund, M., Grevesse, N., Sauval, A. J., \& Scott, P. 2009, ARA\&A, 47, 481 Bekki, K. 2008, MNRAS, 388, L10 Bianchi, S. 2013, A\&A, 552, A89
Bianchi, S., \& Schneider, R. 2007, MNRAS, 378, 973

Bolatto, A. D., Leroy, A. K., Rosolowsky, E., Walter, F., \& Blitz, L. 2008, ApJ, 686, 948

Bolatto, A. D., Wolfire, M., \& Leroy, A. K. 2013, ARA\&A, 51, 207

Bruzual, G., \& Charlot, S. 2003, MNRAS, 344, 1000

Calura, F., Pipino, A., \& Matteucci, F. 2008, A\&A, 479, 669

Cannon, J. M., Skillman, E. D., Garnett, D. R., \& Dufour, R. J. 2002, ApJ, 565, 931

Cannon, J. M., Walter, F., Skillman, E. D., \& van Zee, L. 2005, ApJ, 621, L21

Cardelli, J. A., Clayton, G. C., \& Mathis, J. S. 1989, ApJ, 345, 245

Chabrier, G. 2003, PASP, 115, 763

Chary, R., \& Elbaz, D. 2001, ApJ, 556, 562

Chen, B., Dai, X., Kochanek, C. S., \& Chartas, G. 2013, ApJ, submitted [arXiv: 1306.0008]

Contreras Ramos, R., Annibali, F., Fiorentino, G., et al. 2011, ApJ, 739, 74

Cortese, L., Ciesla, L., Boselli, A., et al. 2012, A\&A, 540, A52

da Cunha, E., Eminian, C., Charlot, S., \& Blaizot, J. 2010, MNRAS, 403, 1894

Daddi, E., et al. 2010, ApJ, 714, L118

Dale, D. A., Helou, G., Neugebauer, G., et al. 2001, AJ, 122, 1736

Dale, D. A., Aniano, G., Engelbracht, C. W., et al. 2012, ApJ, 745, 95

Dole, H., Lagache, G., Puget, J.-L., et al. 2006, A\&A, 451, 417

Draine, B. T., \& Li, A. 2007, ApJ, 657, 810

Draine, B. T., Dale, D. A., Bendo, G., et al. 2007, ApJ, 663, 866

Eales, S., Smith, M. W. L., Auld, R., et al. 2012, ApJ, 761, 168

Ekta, Chengalur, J. N., \& Pustilnik, S. A. 2008, MNRAS, 391, 881

Ekta, B., Pustilnik, S. A., \& Chengalur, J. N. 2009, MNRAS, 397, 963

Engelbracht, C. W., Rieke, G. H., Gordon, K. D., et al. 2008, ApJ, 678, 804

Fazio, G. G., Hora, J. L., Allen, L. E., et al. 2004, ApJS, 154, 10

Fisher, D. B., Bolatto, A. D., Herrera-Camus, R., et al. 2013, Nature, in press [arXiv: 1310.4842$]$

Franceschini, A., Rodighiero, G., \& Vaccari, M. 2008, A\&A, 487, 837

Fumagalli, M., Krumholz, M. R., \& Hunt, L. K. 2010, ApJ, 722, 919

Galametz, M., Madden, S. C., Galliano, F., et al. 2011, A\&A, 532, A56

Genzel, R., Tacconi, L. J., Combes, F., et al. 2012, ApJ, 746, 69

Gil de Paz, A., Madore, B. F., \& Pevunova, O. 2003, ApJS, 147, 29

Gruppioni, C., Pozzi, F., Andreani, P., et al. 2010, A\&A, 518, L27

Guilloteau, S., Lucas, R. 2000, Imaging at Radio through Submillimeter Wavelengths, ASP Conf. Ser., 217, 299

Hauser, M. G., \& Dwek, E. 2001, ARA\&A, 39, 249

Herrera-Camus, R., Fisher, D. B., Bolatto, A. D., et al. 2012, ApJ, 752, 112

Hirashita, H., Tajiri, Y. Y., \& Kamaya, H. 2002, A\&A, 388, 439

Houck, J. R., Charmandaris, V., Brandl, B. R., et al. 2004a, ApJS, 154, 211

Houck, J. R., Roellig, T. L., van Cleve, J., et al. 2004b, ApJS, 154, 18

Hunter, D. A., \& Thronson, H. A., Jr. 1995, ApJ, 452, 238

Hunt, L. K., Vanzi, L., \& Thuan, T. X. 2001, A\&A, 377, 66

Hunt, L. K., Thuan, T. X., \& Izotov, Y. I. 2003, ApJ, 588, 281

Hunt, L. K., Dyer, K. K., Thuan, T. X., \& Ulvestad, J. S. 2004, ApJ, 606, 853

Hunt, L., Bianchi, S., \& Maiolino, R. 2005a, A\&A, 434, 849

Hunt, L. K., Dyer, K. K., \& Thuan, T. X. 2005b, A\&A, 436, 837

Hunt, L., Magrini, L., Galli, D., et al. 2012, MNRAS, 427, 906

Ivezic, Z., \& Elitzur, M. 1997, MNRAS, 287, 799

Izotov, Y. I., Chaffee, F. H., Foltz, C. B., et al. 1999, ApJ, 527, 757

Izotov, Y. I., Thuan, T. X., \& Guseva, N. G. 2005, ApJ, 632, 210

Izotov, Y. I., Thuan, T. X., \& Stasińska, G. 2007, ApJ, 662, 15

James, A., Dunne, L., Eales, S., \& Edmunds, M. G. 2002, MNRAS, 335, 753

Johnson, K. E., Hunt, L. K., \& Reines, A. E. 2009, AJ, 137, 3788

Kennicutt, R. C., Jr. 1998, ARA\&A, 36, 189

Kennicutt, R. C., Calzetti, D., Aniano, G., et al. 2011, PASP, 123, 1347

Krumholz, M. R. 2012, ApJ, 759, 9

Lelli, F., Verheijen, M., Fraternali, F., \& Sancisi, R. 2012, A\&A, 537, A72

Leroy, A., Cannon, J., Walter, F., Bolatto, A., \& Weiss, A. 2007, ApJ, 663, 990

Leroy, A. K., Bolatto, A., Gordon, K., et al. 2011, ApJ, 737, 12

Magdis, G. E., Daddi, E., Elbaz, D., et al. 2011, ApJ, 740, L15

Makovoz, D., \& Marleau, F. R. 2005, PASP, 117, 1113

Mao, R.-Q., Schulz, A., Henkel, C., et al. 2010, ApJ, 724, 1336

McMullin, J. P., Waters, B., Schiebel, D., Young, W., Golap, K. 2007, Astronomical Data Analysis Software and Systems XVI, ASP Conf. Ser., 376,127

Meier, D. S., Turner, J. L., Crosthwaite, L. P., \& Beck, S. C. 2001, AJ, 121, 740

Nikutta, R. 2012, Ph.D. Thesis, University of Kentucky, USA, http: //uknowledge.uky.edu/physastron_etds/8/

Nozawa, T., Kozasa, T., Habe, A., et al. 2007, ApJ, 666, 955

Onodera, S., Kuno, N., Tosaki, T., et al. 2010, ApJ, 722, L127

Osterbrock, D. E., \& Ferland, G. J. 2006, Astrophysics of gaseous nebulae and active galactic nuclei, 2nd edn. (Sausalito, CA: University Science Books) 
Ott, S. 2010, ASP Conf. Ser., 434, 139

Papaderos, P., Izotov, Y. I., Fricke, K. J., Thuan, T. X., \& Guseva, N. G. 1998, A\&A, 338, 43

Papaderos, P., Izotov, Y. I., Thuan, T. X., et al. 2002, A\&A, 393, 461

Pilyugin, L. S., \& Thuan, T. X. 2005, ApJ, 631, 231

Plante, S., \& Sauvage, M. 2002, AJ, 124, 1995

Poglitsch, A., Waelkens, C., Geis, N., et al. 2010, A\&A, 518, L2

Pustilnik, S. A., Brinks, E., Thuan, T. X., Lipovetsky, V. A., \& Izotov, Y. I. 2001, AJ, 121,1413

Reines, A. E., Johnson, K. E., \& Hunt, L. K. 2008, AJ, 136, 1415

Rémy-Ruyer, A., Madden, S. C., Galliano, F., et al. 2013, A\&A, 557, A95

Rieke, G. H., Young, E. T., Engelbracht, C. W., et al. 2004, ApJS, 154, 25

Roussel, H. 2013, PASP, 125, 1126

Rowlands, K., Dunne, L., Maddox, S., et al. 2012, MNRAS, 419, 2545

Santini, P., Maiolino, R., Magnelli, B., et al. 2010, A\&A, 518, L154
Sargent, M. T., Daddi, E., Béthermin, M., et al. 2013, ApJ, submitted [arXiv: 1303.4392]

Schneider, R., Ferrara, A., \& Salvaterra, R. 2004, MNRAS, 351, 1379

Skibba, R. A., Engelbracht, C. W., Dale, D., et al. 2011, ApJ, 738, 89

Thuan, T. X., \& Izotov, Y. I. 1997a, ApJ, 489, 623

Thuan, T. X., Izotov, Y. I., \& Lipovetsky, V. A. 1997b, ApJ, 477, 661

Todini, P., \& Ferrara, A. 2001, MNRAS, 325, 726

Vader, J. P., Frogel, J. A., Terndrup, D. M., \& Heisler, C. A. 1993, AJ, 106, 1743

Valiante, R., Schneider, R., Bianchi, S., \& Andersen, A. C. 2009, MNRAS, 397, 1661

Vanzi, L., Hunt, L. K., Thuan, T. X., \& Izotov, Y. I. 2000, A\&A, 363, 493

Verley, S., Corbelli, E., Giovanardi, C., \& Hunt, L. K. 2010, A\&A, 510, A64

Wang, R., Carilli, C. L., Wagg, J., et al. 2008, ApJ, 687, 848

Weingartner, J. C., \& Draine, B. T. 2001, ApJ, 548, 296

Wu, Y., Charmandaris, V., Hunt, L. K., et al. 2007, ApJ, 662, 952 